\title{
On the Irrotational Flow of Incompressible Ideal Fluid in a Circular Domain with Free Surface
}

\author{
Dedicated to Professor S. Irie for his 70th birthday
}

By

\section{Tatsuo IGUCH*}

\section{§1. Introduction}

We are concerned with a free boundary problem for the two-dimensional and irrotational flow of incompressible ideal fluid around an obstacle. We assume that the domain occupied by the fluid is surrounded by two closed Jordan curves. The inner curve is the boundary of the obstacle, while the outer curve is a free boundary. We take the gravitation due to the obstacle into account. Physically, this obstacle represents the earth and the fluid represents the ocean or the atmosphere. This type of problem was already considered by Okamoto [3], [4], [5], [6]. He studied stationary solutions as a bifurcation problem and the stability of trivial stationary solutions. Up to the present day, however, there is no existence theorem of the corresponding Cauchy problem.

On the other hand, Nalimov [2] investigated the Cauchy problem for surface waves of different type from the above one. In his formulation, the domain occupied by the fluid has infinite extent and depth. He showed that the problem is well-posed in suitable Sobolev spaces of finite smoothness under the restriction that the initial data are close to the equilibrium rest state. Then, Yosihara [7] extended the Nalimov's result to the case of presence of an almost flat bottom. It is worth while mentioning that they used the Lagrangian coordinates to reduce the problem to an equivalent one on the free surface. Moreover, there was a cancellation in quasi-linearization of the

Communicated by H. Okamoto, June 10, 1997.

Revised April 7, 1998.

1991 Mathematics Subject Classification(s): 76B15, 35Q35, 35R35

* Graduate School of Mathematics, Kyushu University Hakozaki, Fukuoka 812-8581, Japan 
reduced system of non-linear equations. This cancellation was essential to show the well-posedness of the Cauchy problem.

In the present paper, on the basis of their analysis we shall investigate the well-posedness of the Cauchy problem for our case. In section 2 we formulate the problem, transform it into an equivalent one by using the Lagrangian coordinates and give the statements of main theorems. In section 3 some notations, definitions and preliminary lemmas are presented. In section 4 we give the explicit form of the operator $K$, which appears in the transformed problem. In section 5 the properties of $K$ are investigated. To this end, we study a certain class of integral operators. In section 6 the transformed problem is reduced to a quasi-linear system. In this reduction a cancellation appears as in [2], [7]. In sections 7 and 8, we give the proofs of the main theorems.

\section{$\S 2 . \quad$ Formulation and Results}

We assume that the domain $\Omega_{t}$ occupied by the fluid at time $t \geq 0$, the free surface $\Gamma_{t}$ and the rigid boundary $\Sigma$ of an obstacle are of the following forms

$$
\begin{aligned}
& \Omega_{t}=\left\{z=r \mathrm{e}^{i \theta} ; r_{0}(1+b(\theta))<r<r_{1}(1+\gamma(t, \theta)), \quad 0 \leq \theta<2 \pi\right\}, \\
& \Gamma_{t}=\left\{z=r \mathrm{e}^{i \theta} ; r=r_{1}(1+\gamma(t, \theta)), \quad 0 \leq \theta<2 \pi\right\}, \\
& \Sigma=\left\{z=r \mathrm{e}^{i \theta} ; r=r_{0}(1+b(\theta)), \quad 0 \leq \theta<2 \pi\right\},
\end{aligned}
$$

where $r_{0}$ and $r_{1}$ are positive constants satisfying the relation $r_{0}<r_{1}$ and $b$ is a given function, while $\gamma$ is the unknown. Here and in what follows, the two-dimensional Euclidean space $\mathbf{R}^{2}$ is identified with the complex plane $\mathbb{C}$ in the usual manner, and this identification is used not only for the spatial variables but also for unknown vectors. The motion of the fluid is described by the velocity $v=\left(v_{1}, v_{2}\right)$ and the pressure $p$ satisfying the equations

$$
\begin{aligned}
& \rho\left(\frac{\partial v}{\partial t}+(v \cdot \nabla) v\right)+\nabla p=\rho g \nabla \frac{1}{|z|} \quad \text { in } \quad \Omega_{t}, \quad t>0 \\
& \frac{\partial v_{1}}{\partial z_{1}}+\frac{\partial v_{2}}{\partial z_{2}}=0, \quad \frac{\partial v_{2}}{\partial z_{1}}-\frac{\partial v_{1}}{\partial z_{2}}=0 \quad \text { in } \quad \Omega_{t}, \quad t>0,
\end{aligned}
$$

where $\nabla=\left(\partial / \partial z_{1}, \partial / \partial z_{2}\right), v \cdot \nabla=v_{1}\left(\partial / \partial z_{1}\right)+v_{2}\left(\partial / \partial z_{2}\right), \rho$ is a constant density, $g$ is a gravitational constant and it is assumed that the center of gravity is located at the origin. The dynamical and kinematical boundary conditions on the free surface $\Gamma_{t}$ are given by 


$$
p=p_{0} \quad \text { on } \quad \Gamma_{t}, \quad t>0
$$

and

$$
\left(\frac{\partial}{\partial t}+v \cdot \nabla\right)\left(r_{1}(1+\gamma)-r\right)=0 \quad \text { on } \quad \Gamma_{t}, \quad t>0
$$

respectively, where $p_{0}$ is an external pressure assumed to be constant. The boundary condition on the rigid boundary $\Sigma$ is given by

$$
v \cdot n_{b}=0 \quad \text { on } \quad \Sigma, \quad t>0
$$

where $n_{b}$ is the unit outward normal vector to $\Sigma$. Finally, we impose the initial conditions

$$
\gamma(0, \theta)=\gamma_{0}(\theta), \quad v(0, z)=v_{0}(z)
$$

The initial velocity $v_{0}$ is assumed to satisfy the compatibility conditions (2.2) and (2.5).

We transform the above system of equations using the Lagrangian coordinates. Let

$$
\Gamma_{t}: z=r_{1}\left(1+X_{2}(t, \theta)\right) \mathrm{e}^{i\left(\theta+X_{1}(t, \theta)\right)}, \quad 0 \leq \theta<2 \pi
$$

be the parameter-representation of the free surface such that

$$
\frac{\partial}{\partial t}\left(r_{1}\left(1+X_{2}(t, \theta)\right) \mathrm{e}^{i\left(\theta+X_{1}(t, \theta)\right)}\right)=v\left(t, r_{1}\left(1+X_{2}(t, \theta)\right) \mathrm{e}^{i\left(\theta+X_{1}(t, \theta)\right)}\right) .
$$

Then $X=\left(X_{1}, X_{2}\right)$ satisfy the following system of equations:

$$
\begin{gathered}
\left(1+X_{2}\right)\left(1+X_{1 \theta}\right)\left(\left(1+X_{2}\right) X_{1 t t}+2 X_{1 t} X_{2 t}\right) \\
+X_{2 \theta}\left(X_{2 t t}-\left(1+X_{2}\right) X_{1 t}^{2}+\kappa\left(1+X_{2}\right)^{-2}\right)=0 \quad \text { for } \quad t>0 \\
X_{2 t}=K\left(1+X_{2}\right) X_{1 t} \quad \text { for } \quad t>0 \\
X=U, \quad X_{1 t}=V \quad \text { at } \quad t=0
\end{gathered}
$$

where $\kappa=g / r_{1}^{3}$ and $K=K(X, b, \beta)$ is a linear operator depending on $X, b$ and $\beta=r_{1} / r_{0}$. These are derived as follows. Differentiating (2.8) with respect to $t$ and using (2.1) we see that 


$$
\begin{array}{r}
\left(X_{2 t t}-\left(1+X_{2}\right) X_{1 t}^{2}+i\left(2 X_{2 t} X_{1 t}+\left(1+X_{2}\right) X_{1 t t}\right)\right) \mathrm{e}^{i\left(\theta+X_{1}\right)} \\
=-\kappa\left(1+X_{2}\right)^{-2} \mathrm{e}^{i\left(\theta+X_{1}\right)}-\left.\left(\rho r_{1}\right)^{-1} \nabla p\right|_{\Gamma_{t}} .
\end{array}
$$

On the other hand, differentiating (2.3) with respect to the tangential direction $\theta$ we get

$$
\left.\left(X_{2 \theta}+i\left(1+X_{2}\right)\left(1+X_{1 \theta}\right)\right) \mathrm{e}^{i\left(\theta+X_{1}\right)} \cdot \nabla p\right|_{\Gamma_{t}}=0 .
$$

Eliminating $\left.\nabla p\right|_{\Gamma_{t}}$ from these two relations, we obtain (2.9). (2.2) and (2.5) imply that the normal and the tangential components of the velocity $v$ on the free surface are not independent. (2.10) represents this relation, which is explained in section 4 in full detail. Finally, the initial data $U$ and $V$ are calculated from $\gamma_{0}$ and $v_{0}$ by utilizing (2.8).

The following is one of our main results in the present paper (The function spaces used there are described in section 3).

Theorem 1. For any $\beta>1$ and $\kappa>0$, there exists a small positive constant $\delta_{1}=\delta_{1}(\beta, \kappa)$ depending only on $\beta$ and $\kappa$ such that if $s \geq 4+1 / 2$ and

$$
U \in H^{s+1 / 2}, \quad V, b \in H^{s}, \quad\|U\|_{3} \leq \delta_{1}, \quad\|V\|_{2} \leq \delta_{1}, \quad\|b\|_{3} \leq \delta_{1},
$$

then problem (2.9)-(2.11) has a unique solution $X$ on some time interval $[0, T]$ satisfying

$$
X \in \bigcap_{j=1}^{4} C^{j}\left([0, T] ; H^{s+1 / 2-j / 2}\right)
$$

Moreover, let $X^{(n)}, n=1,2, \ldots$ be the solutions of (2.9)-(2.11) with initial data $\left(U^{(n)}, V^{(n)}\right)$ satisfying $(2.12)$ and $\left(U^{(n)}, V^{(n)}\right) \rightarrow(U, V)$ in $H^{s+1 / 2} \times H^{s}$ as $n \rightarrow \infty$. Then it holds that $X^{(n)} \rightarrow X$ in $\bigcap_{j=1}^{4} C^{j}\left([0, T] ; H^{s+1 / 2-j / 2}\right)$ as $n \rightarrow \infty$.

Remark 2.1. Once the solution $X$ of problem (2.9)-(2.11) has been determined, we can easily obtain the solution of problem (2.1)-(2.6) in the following way. In view of (2.7), $\gamma$ is obtained by the implicit function theorem. We solve the boundary value problem (2.2) with the boundary conditions (2.5) and $\left.v\right|_{\Gamma_{t}}=\left(r_{1}\left(1+X_{2}\right) \mathrm{e}^{i\left(\theta+X_{1}\right)}\right)_{t}$. Although these boundary conditions are overdetermined, the problem can be solvable because of (2.10). Then $p$ is determined by (2.1) and (2.3) due to (2.9). 
Next, we restrict ourselves to the case $b=0$, that is, the rigid boundary $\Sigma$ is just a circle with a radius $r_{0}$. In this case, problem (2.1)-(2.5) has a circulating stationary solution of the form

$$
\left\{\begin{array}{l}
\gamma=0, \quad v(z)=-r_{1} a\left(\frac{\partial}{\partial z_{2}}-i \frac{\partial}{\partial z_{1}}\right) \log |z| \\
p(z)=p_{0}+\rho g\left(\frac{1}{|z|}-\frac{1}{r_{1}}\right)-\frac{\rho r_{1}^{2} a^{2}}{2}\left(\frac{1}{|z|^{2}}-\frac{1}{r_{1}^{2}}\right)
\end{array}\right.
$$

where $a$ is a real parameter, which denotes the speed of the flow on the free surface. In our Lagrangian coordinates, the above solution corresponds to $X=(\alpha t, 0)$ with $\alpha=a / r_{1}$. Now, we proceed to investigate the well-posedness for the Cauchy problem (2.1)-(2.6) around the stationary solution (2.14). To this end, we replace $X_{1}$ by $X_{1}+\alpha t$ in (2.9) and (2.10). Then, we obtain

$$
\begin{gathered}
\left(1+X_{2}\right)\left(1+X_{1 \theta}\right)\left(\left(1+X_{2}\right) X_{1 t t}+2\left(\alpha+X_{1 t}\right) X_{2 t}\right) \\
+X_{2 \theta}\left(X_{2 t t}-\left(1+X_{2}\right)\left(\alpha+X_{1 t}\right)^{2}+\kappa\left(1+X_{2}\right)^{-2}\right)=0 \quad \text { for } t>0 \\
X_{2 t}=K\left(1+X_{2}\right)\left(\alpha+X_{1 t}\right) \quad \text { for } \quad t>0
\end{gathered}
$$

where $K=K(X, 0, \beta)$ is the same operator as in (2.10) with $b=0$.

Before stating our results concerning the initial value problem for (2.15) and (2.16), we consider the linearized equations:

$$
\begin{gathered}
X_{1 t t}+2 \alpha X_{2 t}+\left(\kappa-\alpha^{2}\right) X_{2 \theta}=0, \\
X_{2 t}=K_{0} X_{1 t}+2 \alpha K_{0} X_{2}
\end{gathered}
$$

(for (2.18), see section 4). These imply the equation

$$
X_{2 t t}+\left(\kappa-\alpha^{2}\right) K_{0} X_{2 \theta}=0 .
$$

This equation for $X_{2}$ can be easily analyzed and we see that the initial value problem for (2.19) is well-posed in the class $X_{2} \in \bigcap_{j=0}^{2} C^{j}\left([0, T] ; H^{s+1 / 2-j / 2}\right)$ if $\kappa>\alpha^{2}$, and that the problem is ill-posed if $\alpha^{2}>\kappa$. Since parameters $\alpha$ and $\kappa$ represent the speed of the flow and the strength of the gravity, respectively, these facts express in a physical point of view that if the gravity is stronger than the flow, then the problem is well-posed. On the contrary, if the flow 
is stronger than the gravity, then the problem becomes ill-posed. For the non-linear problem, the same statements are valid and we obtain the following results.

Theorem 2. Suppose that $\beta>1, \alpha, \kappa \in \mathbf{R}^{1}$ and $\kappa>\alpha^{2}$. There exists a small positive constant $\delta_{2}=\delta_{2}(\beta, \alpha, \kappa)$ depending only on $\beta, \alpha$ and $\kappa$ such that if $s \geq 4+1 / 2$ and

$$
U \in H^{s+1 / 2}, \quad V \in H^{s}, \quad\|U\|_{3} \leq \delta_{2}, \quad\|V\|_{2} \leq \delta_{2},
$$

then problem (2.15), (2.16) and (2.11) has a unique solution $X$ on some time interval $[0, T]$ satisfying (2.13). Moreover, the continuous dependence of solutions on the initial data is also valid in the same sense as in Theorem 1.

Theorem 3. Suppose that $\beta>1, \alpha, \kappa \in \mathbf{R}^{1}$ and $\alpha^{2}>\kappa$. Then, problem (2.15), (2.16) and (2.11) is not well-posed. More precisely, the following statement is not true: There exist a large number $N$ and a positive constant $T$ such that for any number $n \geq N$, problem (2.15), (2.16) and (2.11) with initial data

$$
U=0, \quad V=\mathrm{e}^{-n^{1 / 4}+i n \theta}
$$

has a unique solution $X=X^{(n)}$ satisfying

$$
X^{(n)} \rightarrow 0 \quad \text { in } \quad C^{1}\left([0, T] ; H^{4+1 / 2}\right) \text { as } n \rightarrow \infty .
$$

Remark 2.2. The initial data in (2.20) are smooth functions and converge to zero in the Sobolev space of any order. Hence, Theorem 3 implies that the well-posedness for the Cauchy problem (2.15), (2.16) and (2.11) does not hold in the case $\alpha^{2}>\kappa$ even if we assume that the initial data are sufficiently small in suitable Sobolev spaces.

Remark 2.3. The assumptions that the initial data are small in Theorems 1 and 2 can not be removed in general, because the solution of (2.15) and (2.16) with $\alpha=\alpha_{1}+\alpha_{2}$ and (2.11) is also the solution of (2.15) and (2.16) with $\alpha=\alpha_{1}$ and (2.11) with $V$ replaced by $V+\alpha_{2}$, and we have Theorem 3 .

\section{§3. Preliminaries}

For a real number $s$ we denote by $H^{s}$ the usual Sobolev space of $2 \pi$-periodic functions on $\mathbf{R}^{1}$ equipped with the norm 


$$
\|f\|_{s}=\left(2 \pi \sum_{n=-\infty}^{\infty}(1+|n|)^{2 s}\left|f_{n}\right|^{2}\right)^{1 / 2}
$$

where $f_{n}$ is the $n$-th Fourier coefficient of $f$

$$
f_{n}=\frac{1}{2 \pi} \int_{0}^{2 \pi} f(\theta) \mathrm{e}^{-i n \theta} d \theta, \quad n=0, \pm 1, \pm 2, \ldots
$$

For an integer $j \geq 0$ and $0<T<\infty$ we say that $f \in C^{j}([0, T] ; X)$ if $f$ is a function of $C^{j}$-class on the closed interval $[0, T]$ with the value in a Banach space $X$. A pseudo-differential operator $P(D), D=-i \partial / \partial \theta$, with a symbol $P(n)$ is defined by

$$
P(D) f(\theta)=\sum_{n=-\infty}^{\infty} P(n) f_{n} \mathrm{e}^{i n \theta} \quad \text { for } \quad f(\theta)=\sum_{n=-\infty}^{\infty} f_{n} \mathrm{e}^{i n \theta}
$$

We define the pseudo-differential operator $K_{0}$ and the symbol sgnn by

$$
K_{0}=-i \frac{\beta^{2 D}-1}{\beta^{2 D}+1} \quad \text { and } \quad \operatorname{sgn} n= \begin{cases}n /|n| & \text { if } n \neq 0 \\ 0 & \text { if } n=0\end{cases}
$$

respectively. For operators $A$ and $B$, the commutator of $A$ and $B$ is denoted by $[A, B]=A B-B A$. Throughout this paper, the symbol $C$ denotes various positive constants, which are different in different lines and $C=C(a, b, \ldots)$ means that $C$ depends on $a, b, \ldots$ For any $\theta \in \mathbf{R}^{1}$, we define the translation operator $T_{\theta}$ by $\left(T_{\theta} f\right)(\varphi)=f(\theta+\varphi)$.

Lemma 3.1. For $0<r<1$, there exists a constant $C_{r}>0$ depending only on $r$ such that for any $f \in H^{r}$

$$
C_{r}^{-1} \sum_{n=-\infty}^{\infty}|n|^{2 r}\left|f_{n}\right|^{2} \leq \int_{0}^{2 \pi} \int_{0}^{2 \pi} \frac{|f(\varphi)-f(\theta)|^{2}}{\left|\mathrm{e}^{i \varphi}-\mathrm{e}^{i \theta}\right|^{1+2 r}} d \theta d \varphi \leq C_{r} \sum_{n=-\infty}^{\infty}|n|^{2 r}\left|f_{n}\right|^{2} .
$$

Moreover, $\|f\|_{s+r}$ is equivalent to the norm

$$
\|f\|_{s}+\left(\int_{0}^{2 \pi} \frac{\left\|T_{\theta} f-f\right\|_{s}^{2}}{\left|\mathrm{e}^{i \theta}-1\right|^{1+2 r}} d \theta\right)^{1 / 2},
$$

where $s$ is an arbitrary real number and $f \in H^{s+r}$.

Proof. Using Parseval's formula, we see that 


$$
\begin{aligned}
\int_{0}^{2 \pi} \int_{0}^{2 \pi} \frac{|f(\varphi)-f(\theta)|^{2}}{\left|\mathrm{e}^{i \varphi}-\mathrm{e}^{i \theta}\right|^{1+2 r}} d \theta d \varphi & =2 \pi \sum_{n=-\infty}^{\infty}\left|f_{n}\right|^{2} \int_{0}^{2 \pi} \frac{\left|\mathrm{e}^{i n \theta}-1\right|^{2}}{\left|\mathrm{e}^{i \theta}-1\right|^{1+2 r}} d \theta \\
& =4^{2-r} \pi \sum_{n=-\infty}^{\infty}\left|f_{n}\right|^{2} \int_{0}^{\pi / 2} \frac{(\sin n \theta)^{2}}{(\sin \theta)^{1+2 r}} d \theta
\end{aligned}
$$

Since $2 \theta / \pi \leq \sin \theta \leq \theta$ for $0 \leq \theta \leq \pi / 2$, it holds for any integer $n \neq 0$ that

$$
\int_{0}^{\pi / 2} \frac{(\sin n \theta)^{2}}{(\sin \theta)^{1+2 r}} d \theta \geq \int_{0}^{\pi / 2|n|} \frac{(2 n \theta / \pi)^{2}}{\theta^{1+2 r}} d \theta=\frac{1}{2(1-r)}\left(\frac{2|n|}{\pi}\right)^{2 r}
$$

and

$$
\int_{0}^{\pi / 2} \frac{(\sin n \theta)^{2}}{(\sin \theta)^{1+2 r}} d \theta \leq \int_{0}^{\pi / 2|n|} \frac{(n \theta)^{2}}{(2 \theta / \pi)^{1+2 r}} d \theta+\int_{\pi / 2|n|}^{\pi / 2} \frac{d \theta}{(2 \theta / \pi)^{1+2 r}} \leq \frac{\pi^{3}}{16 r(1-r)}|n|^{2 r} .
$$

These imply the first assertion. For the second one, it is sufficient to note that

$$
\left\|T_{\theta} f-f\right\|_{s}^{2}=2 \pi \sum_{n=-\infty}^{\infty}(1+|n|)^{2 s}\left|f_{n}\right|^{2}\left|\mathrm{e}^{i n \theta}-1\right|^{2}
$$

because of the above estimates. The proof is complete.

Lemma 3.2. Let $\beta>1$ and $s \geq 0$. For any $f \in H^{0}$ we have

$$
\left\|\left(i \operatorname{sgn} D+K_{0}\right) f\right\|_{s} \leq C\|f\|_{0}, \quad\left\|\left(1+K_{0}^{2}\right) f\right\|_{s} \leq C\|f\|_{0},
$$

where $C=C(\beta, s)>0$.

Proof. In view of the inequalities

$$
\left|i \operatorname{sgn} n-i \frac{\beta^{2 n}-1}{\beta^{2 n}+1}\right| \leq 2 \beta^{-2|n|} \quad \text { and } \quad\left|1+\left(-i \frac{\beta^{2 n}-1}{\beta^{2 n}+1}\right)^{2}\right| \leq 4 \beta^{-2|n|}
$$

we obtain the desired estimates. The proof is complete.

Lemma 3.3. Let $r, t \geq 0$ and $\varepsilon>0$. For $a \in H^{r+t}$ and $f \in H^{1 / 2+\varepsilon-t}$, we have

$$
\|[\operatorname{sgn} D, a] f\|_{r} \leq(2 \pi)^{-1 / 2}(1+1 / \varepsilon)^{1 / 2}\|a\|_{r+t}\|f\|_{1 / 2+\varepsilon-t} .
$$

For the proof of this lemma, we refer to Lemma 1 in [2] or Lemma 2.14 in [7].

When we investigate the properties of $K$, it is convenient to use the operator 
classes $L(r, s ; t)$ and $L_{0}(r, s ; t)$ which were introduced by Yosihara [7]:

Definition 3.1. Suppose that $0 \leq r \leq s$ and $0 \leq t \leq s$. For an operator $M=M(P)$ depending on $P=\left(P_{1}, \ldots, P_{k}\right)$, we write $M(P ; P(J)) \in L(r, s ; t)(J$ is the subset of $\{1, \ldots, k\}, P(J)=\left(P_{j_{1}}, \ldots, P_{j_{1}}\right)$ if $J=\left\{j_{1}, \ldots, j_{l}\right\}$ and $P(J)=0$ if $J$ is empty) if the following condition is fulfilled: There exists $\varepsilon>0$ such that if $P, P^{0} \in H^{s}$ satisfy $\|P(J)\|_{t}, \quad\left\|P^{0}(J)\right\|_{t} \leq \varepsilon$ and $\|P\|_{s},\left\|P^{0}\right\|_{s} \leq c$ for some $c>0$, then $\|M(P) f\|_{s} \leq C\|f\|_{r}$ and $\left\|M(P) f-M\left(P^{0}\right) f\right\|_{s} \leq C\left\|P-P^{0}\right\|_{s}\|f\|_{r}$ for $f \in H^{r}$, where the constant $C$ does not depend on $P$ and $P^{0}$ but on $c$.

We write $M(P ; P(J)) \in L_{0}(r, s ; t)$ if $M(P ; P(J)) \in L(r, s ; t)$ and $\|M(P) f\|_{s}$ $\leq C\|P\|_{s}\|f\|_{r}$ for $f \in H^{r}$.

The following is a slight improvement of Lemma 4.22 in [7].

Lemma 3.4. Suppose that $0 \leq r \leq s$ and $0 \leq t \leq s \leq s_{1}$. Then

1) $L(r, s ; t)$ and $L_{0}(r, s ; t)$ are algebras,

2) $L_{0}(r, s ; t)$ is a two-sided $L(r, s ; t)$-module,

3) If $m$ is smooth in a neighbourhood of $0 \in \mathbf{R}^{k}$, then the operator $M$ defined by $M(P ; P) u=m(P) u$ belongs to $L(s, s ; t)$ for $1 / 2<t \leq s$,

4) If $M(P ; P) \in L_{0}(q, q ; t)$ for any $q \in\left[s, s_{1}\right]$ and $T_{\theta} M(P)=M\left(T_{\theta} P\right) T_{\theta}$ for $\theta \in \mathbf{R}^{1}$, then $M_{1}(P ; P)=(1+M)^{-1} \in L(q, q ; s)$ for any $q \in\left[s, s_{1}\right]$.

\section{§4. Representation of $K$}

Throughout this section the time $t(\geq 0)$ is arbitrarily fixed, so that we simplify $\Omega_{t}, X(t, \theta)$ as $\Omega, X(\theta)$, etc. Assume that the velocity $v$ is of $C^{1}$-class in the domain $\Omega$, continuous up to the boundary $\partial \Omega=\Gamma \cup \Sigma$ and satisfies (2.2), and that $X$ is a $2 \pi$-periodic $C^{1}$-function on $\mathbf{R}^{1}$. These assumptions are always satisfied if we deal with the function spaces (2.13). Recall that the free surface $\Gamma$ and the rigid boundary $\Sigma$ are parameterized as

$$
\left\{\begin{array}{l}
\Gamma: z(\theta)=r_{1}\left(1+X_{2}(\theta)\right) \mathrm{e}^{i\left(\theta+X_{1}(\theta)\right)}, \quad 0 \leq \theta<2 \pi, \\
\Sigma: w(\theta)=r_{0}(1+b(\theta)) \mathrm{e}^{i \theta}, \quad 0 \leq \theta<2 \pi .
\end{array}\right.
$$

Put

$$
\left\{\begin{array}{l}
F(z)=v_{1}(z)-i v_{2}(z), \quad f(\theta)=F(z(\theta)), \quad g(\theta)=F(w(\theta)) \\
V(\theta)=f(\theta) \mathrm{e}^{i\left(\theta+X_{1}(\theta)\right)}, \quad V^{(r)}-i V^{(\theta)}=V \\
W(\theta)=g(\theta) \mathrm{e}^{i \theta}, \quad W^{(r)}-i W^{(\theta)}=W .
\end{array}\right.
$$


Then $F$ is holomorphic in $\Omega$, since (2.2) asserts that $F$ satisfies the Cauchy-Riemann equations. It follows from (2.8) that

$$
V^{(r)}=r_{1} X_{2 t}, \quad V^{(\theta)}=r_{1}\left(1+X_{2}\right) X_{1 t} .
$$

The boundary condition (2.5) on $\Sigma$ can be rewritten in the form

$$
W^{(r)}=(1+b)^{-1} b^{\prime} W^{(\theta)} .
$$

Taking $z_{0}=z(\theta) \in \Gamma$, applying the Cauchy integral formula to $F$ in the domain $\Omega(r)=\left\{z ;\left|z-z_{0}\right|>r\right\} \cap \Omega$ and letting $r \rightarrow+0$, we obtain

$$
0=\frac{1}{2 \pi i} \oint_{\partial \Omega(r)} \frac{F(z)}{z-z_{0}} d z=\frac{-\pi i}{2 \pi i} F\left(z_{0}\right)+\frac{1}{2 \pi i} \text { p.v. } \int_{\Gamma} \frac{F(z)}{z-z_{0}} d z-\frac{1}{2 \pi i} \int_{\Sigma} \frac{F(z)}{z-z_{0}} d z .
$$

Transforming the coordinates yields that

$$
f(\theta)=\frac{1}{\pi i} \text { p.v. } \int_{0}^{2 \pi} \frac{f(\varphi)}{z(\varphi)-z(\theta)} \frac{d z(\varphi)}{d \varphi} d \varphi-\frac{1}{\pi i} \int_{0}^{2 \pi} \frac{g(\varphi)}{w(\varphi)-z(\theta)} \frac{d w(\varphi)}{d \varphi} d \varphi .
$$

The integral kernels can be rewritten in the form

$$
\begin{gathered}
\frac{1}{z(\varphi)-z(\theta)} \frac{d z(\varphi)}{d \varphi}=\frac{i \mathrm{e}^{i \varphi}}{\mathrm{e}^{i \varphi}-\mathrm{e}^{i \theta}}+\frac{\partial}{\partial \varphi} \log \left(\frac{\left(1+X_{2}(\varphi)\right) \mathrm{e}^{i\left(\varphi+X_{1}(\varphi)\right)}-\left(1+X_{2}(\theta)\right) \mathrm{e}^{i\left(\theta+X_{1}(\theta)\right)}}{\mathrm{e}^{i \varphi}-\mathrm{e}^{i \theta}}\right), \\
\frac{1}{w(\varphi)-z(\theta)} \frac{d w(\varphi)}{d \varphi}=\frac{i \mathrm{e}^{i \varphi}}{\mathrm{e}^{i \varphi}-\beta \mathrm{e}^{i \theta}}+\frac{\partial}{\partial \varphi} \log \left(\frac{(1+b(\varphi)) \mathrm{e}^{i \varphi}-\beta\left(1+X_{2}(\theta)\right) \mathrm{e}^{i\left(\theta+X_{1}(\theta)\right)}}{\mathrm{e}^{i \varphi}-\beta \mathrm{e}^{i \theta}}\right) .
\end{gathered}
$$

Substituting these, $f=V \mathrm{e}^{-i\left(\theta+X_{1}\right)}$ and $g=W \mathrm{e}^{-i \theta}$ into (4.3) and making use of the integration by parts, we can get

$$
\begin{array}{r}
V(\theta)=-\frac{i}{\pi} \text { p.v. } \int_{0}^{2 \pi} \frac{i \mathrm{e}^{i \theta}}{\mathrm{e}^{i \varphi}-\mathrm{e}^{i \theta}} V(\varphi) d \varphi+\frac{i}{\pi} \int_{0}^{2 \pi} \frac{i \mathrm{e}^{i \theta}}{\mathrm{e}^{i \varphi}-\beta \mathrm{e}^{i \theta}} W(\varphi) d \varphi \\
-\frac{1}{\pi} \int_{0}^{2 \pi} \mathrm{e}^{i \theta-i X_{1}(\varphi)} \frac{\mathrm{e}^{i X_{1}(\varphi)}-\mathrm{e}^{i X_{1}(\theta)}}{\mathrm{e}^{i \varphi}-\mathrm{e}^{i \theta}} V(\varphi) d \varphi+\frac{1}{\pi} \int_{0}^{2 \pi} \mathrm{e}^{i \theta} \frac{1-\mathrm{e}^{i X_{1}(\theta)}}{\mathrm{e}^{i \varphi}-\beta \mathrm{e}^{i \theta}} W(\varphi) d \varphi \\
+\frac{1}{\pi} \int_{0}^{2 \pi} \mathrm{e}^{i\left(\theta+X_{1}(\theta)\right)-i\left(\varphi+X_{1}(\varphi)\right)} \log \left(\frac{\left(1+X_{2}(\varphi)\right) \mathrm{e}^{i\left(\varphi+X_{1}(\varphi)\right)}-\left(1+X_{2}(\theta)\right) \mathrm{e}^{i\left(\theta+X_{1}(\theta)\right)}}{\mathrm{e}^{i \varphi}-\mathrm{e}^{i \theta}}\right) \\
\times\left(\left(1+\frac{d X_{1}(\varphi)}{d \varphi}\right) V(\varphi)+i \frac{d V(\varphi)}{d \varphi}\right) d \varphi
\end{array}
$$




$$
\begin{gathered}
-\frac{1}{\pi} \int_{0}^{2 \pi} \mathrm{e}^{i\left(\theta+X_{1}(\theta)\right)-i \varphi} \log \left(\frac{(1+b(\varphi)) \mathrm{e}^{i \varphi}-\beta\left(1+X_{2}(\theta)\right) \mathrm{e}^{i\left(\theta+X_{1}(\theta)\right)}}{\mathrm{e}^{i \varphi}-\beta \mathrm{e}^{i \theta}}\right) \\
\times\left(W(\varphi)+i \frac{d W(\varphi)}{d \varphi}\right) d \varphi .
\end{gathered}
$$

Define the projection $P_{0}$ by

$$
P_{0} f=\frac{1}{2 \pi} \int_{0}^{2 \pi} f(\theta) d \theta \quad\left(=f_{0} \text { the } 0 \text {-th Fourier coefficient }\right)
$$

It is not difficult to see that

$$
\frac{1}{\pi} \text { p.v. } \int_{0}^{2 \pi} \frac{i \mathrm{e}^{i \theta}}{\mathrm{e}^{i \varphi}-\mathrm{e}^{i \theta}} f(\varphi) d \varphi=\left(i \operatorname{sgn} D-i P_{0}\right) f,
$$

$\frac{1}{\pi} \int_{0}^{2 \pi} \frac{i \mathrm{e}^{i \theta}}{\mathrm{e}^{i \varphi}-\alpha \mathrm{e}^{i \theta}} f(\varphi) d \varphi= \begin{cases}i \operatorname{sgn} D \alpha^{|D|-1} f+i\left(\alpha^{|D|-1}-\alpha^{-1} P_{0}\right) f & \text { if } 0<\alpha<1, \\ i \operatorname{sgn} D \alpha^{-|D|-1} f-i\left(\alpha^{-|D|-1}+\alpha^{-1} P_{0}\right) f & \text { if } \alpha>1 .\end{cases}$

Therefore, by (4.4) we have

$$
\begin{aligned}
\left(1+P_{0}\right) V= & -i(i \operatorname{sgn} D) V+\beta^{-1} P_{0} W+(1+i(i \operatorname{sgn} D)) \beta^{-|D|-1} W \\
& +\left(\left(-A_{1}+A_{5}-A_{10}\right)+i\left(-A_{2}+A_{6}+A_{9}\right)\right) V \\
& +\left(\left(A_{3}-A_{7}+A_{12}\right)+i\left(A_{4}-A_{8}-A_{11}\right)\right) W
\end{aligned}
$$

where

$$
\left\{\begin{array}{l}
\left(A_{j} f\right)(\theta)=\frac{1}{\pi} \int_{0}^{2 \pi} a_{j}(\varphi, \theta) f(\varphi) d \varphi, \quad j=1, \ldots, 8, \\
a_{j}(\varphi, \theta) \text { are real-valued functions, } \\
a_{1}+i a_{2}=\mathrm{e}^{i \theta-i X_{1}(\varphi)} \frac{\mathrm{e}^{i X_{1}(\varphi)}-\mathrm{e}^{i X_{1}(\theta)}}{\mathrm{e}^{i \varphi}-\mathrm{e}^{i \theta}}, \\
a_{3}+i a_{4}=\mathrm{e}^{i \theta} \frac{1-\mathrm{e}^{i X_{1}(\theta)}}{\mathrm{e}^{i \varphi}-\beta \mathrm{e}^{i \theta}}, \\
a_{5}+i a_{6}=\mathrm{e}^{i\left(\theta+X_{1}(\theta)\right)-i\left(\varphi+X_{1}(\varphi)\right)} \\
\times \log \left(\frac{\left(1+X_{2}(\varphi)\right) \mathrm{e}^{i\left(\varphi+X_{1}(\varphi)\right)}-\left(1+X_{2}(\theta)\right) \mathrm{e}^{i\left(\theta+X_{1}(\theta)\right)}}{\mathrm{e}^{i \varphi}-\mathrm{e}^{i \theta}}\right)\left(1+\frac{d X_{1}(\varphi)}{d \varphi}\right) \\
a_{7}+i a_{8}=\mathrm{e}^{i\left(\theta+X_{1}(\theta)\right)-i \varphi} \log \left(\frac{(1+b(\varphi)) \mathrm{e}^{i \varphi}-\beta\left(1+X_{2}(\theta)\right) \mathrm{e}^{i\left(\theta+X_{1}(\theta)\right)}}{\mathrm{e}^{i \varphi}-\beta \mathrm{e}^{i \theta}}\right)
\end{array}\right.
$$




$$
\left\{\begin{array}{l}
\left(A_{j} f\right)(\theta)=\frac{1}{\pi} \int_{0}^{2 \pi} a_{j}(\varphi, \theta) \frac{d f(\varphi)}{d \varphi} d \varphi, \quad j=9, \ldots, 12, \\
a_{j}(\varphi, \theta) \text { are real-valued functions, } \\
a_{9}+i a_{10}=\mathrm{e}^{i\left(\theta+X_{1}(\theta)\right)-i\left(\varphi+X_{1}(\varphi)\right)} \\
\times \log \left(\frac{\left(1+X_{2}(\varphi)\right) \mathrm{e}^{i\left(\varphi+X_{1}(\varphi)\right)}-\left(1+X_{2}(\theta)\right) \mathrm{e}^{i\left(\theta+X_{1}(\theta)\right)}}{\mathrm{e}^{i \varphi}-\mathrm{e}^{i \theta}}\right), \\
a_{11}+i a_{12}=\mathrm{e}^{i\left(\theta+X_{1}(\theta)\right)-i \varphi} \log \left(\frac{(1+b(\varphi)) \mathrm{e}^{i \varphi}-\beta\left(1+X_{2}(\theta)\right) \mathrm{e}^{i\left(\theta+X_{1}(\theta)\right)}}{\mathrm{e}^{i \varphi}-\beta \mathrm{e}^{i \theta}}\right)
\end{array}\right.
$$

Similarly, taking $w_{0}=w(\theta) \in \Sigma$ and proceeding in the same way as above, we obtain

$$
\begin{aligned}
\left(1-P_{0}\right) W= & i(i \operatorname{sgn} D) W-\beta P_{0} V+(1-i(i \operatorname{sgn} D)) \beta^{-|D|+1} V \\
& +\left(\left(A_{13}+A_{17}-A_{22}\right)+i\left(A_{14}+A_{18}+A_{21}\right)\right) V \\
& +\left(\left(-A_{15}+A_{20}\right)-i\left(A_{16}+A_{19}\right)\right) W
\end{aligned}
$$

where

$$
\begin{aligned}
& \left(A_{j} f\right)(\theta)=\frac{1}{\pi} \int_{0}^{2 \pi} a_{j}(\varphi, \theta) f(\varphi) d \varphi, \quad j=13, \ldots, 18 \\
& a_{j}(\varphi, \theta) \text { are real-valued functions, } \\
& a_{13}+i a_{14}=\mathrm{e}^{i \theta} \frac{\mathrm{e}^{-i X_{1}(\varphi)}-1}{\mathrm{e}^{i \varphi}-\beta^{-1} \mathrm{e}^{i \theta}}, \\
& a_{15}+i a_{16}=\mathrm{e}^{i \theta-i \varphi} \log \left(\frac{(1+b(\varphi)) \mathrm{e}^{i \varphi}-(1+b(\theta)) \mathrm{e}^{i \theta}}{\mathrm{e}^{i \varphi}-\mathrm{e}^{i \theta}}\right), \\
& a_{17}+i a_{18}=\mathrm{e}^{i \theta-i\left(\varphi+X_{1}(\varphi)\right)} \\
& \quad \times \log \left(\frac{\left(1+X_{2}(\varphi)\right) \mathrm{e}^{i\left(\varphi+X_{1}(\varphi)\right)}-\beta^{-1}(1+b(\theta)) \mathrm{e}^{i \theta}}{\mathrm{e}^{i \varphi}-\beta^{-1} \mathrm{e}^{i \theta}}\right)\left(1+\frac{d X_{1}(\varphi)}{d \varphi}\right),
\end{aligned}
$$




$$
\left\{\begin{array}{r}
\left(A_{j} f\right)(\theta)=\frac{1}{\pi} \int_{0}^{2 \pi} a_{j}(\varphi, \theta) \frac{d f(\varphi)}{d \varphi} d \varphi, \quad j=19, \ldots, 22, \\
a_{j}(\varphi, \theta) \text { are real-valued functions, } \\
a_{19}+i a_{20}=\mathrm{e}^{i \theta-i \varphi} \log \left(\frac{(1+b(\varphi)) \mathrm{e}^{i \varphi}-(1+b(\theta)) \mathrm{e}^{i \theta}}{\mathrm{e}^{i \varphi}-\mathrm{e}^{i \theta}}\right) \\
a_{21}+i a_{22}=\mathrm{e}^{i \theta-i\left(\varphi+X_{1}(\varphi)\right)} \\
\times \log \left(\frac{\left(1+X_{2}(\varphi)\right) \mathrm{e}^{i\left(\varphi+X_{1}(\varphi)\right)}-\beta^{-1}(1+b(\theta)) \mathrm{e}^{i \theta}}{\mathrm{e}^{i \varphi}-\beta^{-1} \mathrm{e}^{i \theta}}\right)
\end{array}\right.
$$

It follows from the equation (4.6) applied the projection $P_{0}$ that

$$
\begin{aligned}
P_{0} W= & \beta P_{0} V+(\beta / 2) P_{0}\left(\left(A_{1}-A_{5}+A_{10}\right)+i\left(A_{2}-A_{6}-A_{9}\right)\right) V \\
& -(\beta / 2) P_{0}\left(\left(A_{3}-A_{7}+A_{12}\right)+i\left(A_{4}-A_{8}-A_{11}\right)\right) W .
\end{aligned}
$$

This together with (4.9) implies that

$$
\begin{aligned}
W= & i(i \operatorname{sgn} D) W+(1-i(i \operatorname{sgn} D)) \beta^{-|D|+1} V \\
+ & \left\{\left(A_{13}+A_{17}-A_{22}\right)+i\left(A_{14}+A_{18}+A_{21}\right)\right. \\
& \left.+(\beta / 2) P_{0}\left(\left(A_{1}-A_{5}+A_{10}\right)+i\left(A_{2}-A_{6}-A_{9}\right)\right)\right\} V \\
+ & \left\{\left(-A_{15}+A_{20}\right)-i\left(A_{16}+A_{19}\right)\right. \\
& \left.-(\beta / 2) P_{0}\left(\left(A_{3}-A_{7}+A_{12}\right)+i\left(A_{4}-A_{8}-A_{11}\right)\right)\right\} W .
\end{aligned}
$$

The real part of (4.6) and the imaginary part of (4.12) become

$$
\begin{aligned}
\left(1+P_{0}\right) V^{(r)}= & -(i \operatorname{sgn} D) V^{(\theta)}+\beta^{-|D|-1}(i \operatorname{sgn} D) W^{(\theta)} \\
& +\left(\beta^{-1} P_{0}+\beta^{-|D|-1}+A_{3}-A_{7}+A_{12}\right) W^{(r)}+\left(A_{4}-A_{8}-A_{11}\right) W^{(\theta)} \\
& +\left(-A_{1}+A_{5}-A_{10}\right) V^{(r)}+\left(-A_{2}+A_{6}+A_{9}\right) V^{(\theta)}
\end{aligned}
$$

and

$$
\begin{aligned}
W^{(\theta)}= & \beta^{-|D|+1} V^{(\theta)}+\beta^{-|D|+1}(i \operatorname{sgn} D) V^{(r)} \\
& +\left(-(i \operatorname{sgn} D)+A_{16}+A_{19}+(\beta / 2) P_{0}\left(A_{4}-A_{8}-A_{11}\right)\right) W^{(r)} \\
& +\left(-A_{15}+A_{20}-(\beta / 2) P_{0}\left(A_{3}-A_{7}+A_{12}\right)\right) W^{(\theta)} \\
& -\left(A_{14}+A_{18}+A_{21}+(\beta / 2) P_{0}\left(A_{2}-A_{6}-A_{9}\right)\right) V^{(r)}
\end{aligned}
$$




$$
+\left(A_{13}+A_{17}-A_{22}+(\beta / 2) P_{0}\left(A_{1}-A_{5}+A_{10}\right)\right) V^{(\theta)},
$$

respectively. Eliminating $W^{(r)}$ and $W^{(\theta)}$ from these two relations and (4.2), we obtain $V^{(r)}=K V^{(\theta)}$ with

$$
K=K_{0}+K_{1}, \quad K_{1}=B_{2}+B_{1}\left(1-B_{1}\right)^{-1}\left(K_{0}+B_{2}\right),
$$

where

$$
\begin{aligned}
B_{1}= & \left(1+\beta^{-2|D|}\right)^{-1}\left\{\left(-A_{1}+A_{5}-A_{10}\right)\right. \\
+ & \left(\beta^{-|D|-1}(\text { isgn } D) B_{3}+B_{4}\right)\left(1-B_{3}\right)^{-1} \beta^{-|D|+1}(\text { isgn } D) \\
- & \left(\beta^{-|D|-1}(\text { isgn } D)+B_{4}\right)\left(1-B_{3}\right)^{-1} \\
& \left.\times\left(A_{14}+A_{18}+A_{21}+(\beta / 2) P_{0}\left(A_{2}-A_{6}-A_{9}\right)\right)\right\}, \\
B_{2}= & \left(1+\beta^{-2|D|}\right)^{-1}\left\{\left(-A_{2}+A_{6}+A_{9}\right)\right. \\
+ & \left(\beta^{-|D|-1}(i \operatorname{sgn} D) B_{3}+B_{4}\right)\left(1-B_{3}\right)^{-1} \beta^{-|D|+1} \\
+ & \left(\beta^{-|D|-1}(i \operatorname{sgn} D)+B_{4}\right)\left(1-B_{3}\right)^{-1} \\
& \left.\times\left(A_{13}+A_{17}-A_{22}+(\beta / 2) P_{0}\left(A_{1}-A_{5}+A_{10}\right)\right)\right\}, \\
B_{3}= & \left(-(i \operatorname{sgn} D)+A_{16}+A_{19}+(\beta / 2) P_{0}\left(A_{4}-A_{8}-A_{11}\right)\right)(1+b)^{-1} b^{\prime} \\
& -A_{15}+A_{20}-(\beta / 2) P_{0}\left(A_{3}-A_{7}+A_{12}\right), \\
B_{4}= & \left(\beta^{-1} P_{0}+\beta^{-|D|-1}+A_{3}-A_{7}+A_{12}\right)(1+b)^{-1} b^{\prime}+A_{4}-A_{8}-A_{11} .
\end{aligned}
$$

Thus we get (2.10) because of (4.1).

As for (2.16), it is sufficient to note the above arguments and that in such a case, the free surface $\Gamma$ and the rigid boundary $\Sigma$ are parameterized as

$$
\begin{cases}\Gamma: z(\theta)=r_{1}\left(1+X_{2}(\theta)\right) \mathrm{e}^{i\left(\theta+\alpha t+X_{1}(\theta)\right)}, & 0 \leq \theta<2 \pi, \\ \Sigma: w(\theta)=r_{0} \mathrm{e}^{i(\theta+\alpha t)}, & 0 \leq \theta<2 \pi .\end{cases}
$$

Remark 4.1. In the case $b=0$, applying the above arguments to the velocity $v(z)$ of the stationary solution (2.14), we obtain

$$
\left(K\left(1+X_{2}\right)^{-1}\right)(\theta)=0 \quad \text { for } \quad 0 \leq \theta<2 \pi .
$$

\section{§5. Properties of $K$}

In order to investigate the operator $K$ defined by (4.13), it is sufficient to 
consider the integral operators of the forms

$$
(A f)(\theta)=\int_{0}^{2 \pi}\left(\prod_{j=1}^{M} \frac{a_{j}(\theta)-a_{j}(\varphi)}{\mathrm{e}^{i \theta}-\mathrm{e}^{i \varphi}}\right) F\left(\frac{u(\theta)-u(\varphi)}{\mathrm{e}^{i \theta}-\mathrm{e}^{i \varphi}}\right) f(\varphi) d \varphi
$$

and

$$
(A f)(\theta)=\int_{0}^{2 \pi}\left(\prod_{j=1}^{M} \frac{a_{j}(\theta)+b_{j}(\varphi)}{\mathrm{e}^{i \theta}-\beta \mathrm{e}^{i \varphi}}\right) F\left(\frac{u(\theta)+v(\varphi)}{\mathrm{e}^{i \theta}-\beta \mathrm{e}^{i \varphi}}\right) f(\varphi) d \varphi
$$

where $u=\left(u_{1}, \ldots, u_{N}\right), v=\left(v_{1}, \ldots, v_{N}\right), a_{j}, b_{j}, u_{k}, v_{k}$ are complex valued functions, $F$ is holomorphic in a neighbourhood of $\left\{z \in \mathbf{C}^{N} ;|z| \leq 1 / 2\right\}$ and $\beta$ is a positive constant with $\beta \neq 1$. In the study of these integral operators, of course, it is always assumed that

$$
\left|\frac{u(\theta)-u(\varphi)}{\mathrm{e}^{i \theta}-\mathrm{e}^{i \varphi}}\right| \leq \frac{1}{2} \quad \text { and } \quad\left|\frac{u(\theta)+v(\varphi)}{\mathrm{e}^{i \theta}-\beta \mathrm{e}^{i \varphi}}\right| \leq \frac{1}{2} \quad \text { for } \quad \theta, \varphi \in \mathbf{R}^{1} .
$$

To begin with, we construct estimates for some integrals. For simplicity, we introduce the notation

$$
\tilde{a}(\theta, \varphi)=\frac{a(\theta)-a(\varphi)}{\mathrm{e}^{i \theta}-\mathrm{e}^{i \varphi}}
$$

Lemma 5.1. Let $k$ be a non-negative integer, $s \in \mathbf{R}^{1}, \varepsilon>0$ and $A$ the integral operator of the form

$$
(A f)(\theta)=\int_{0}^{2 \pi}\left(\left(\frac{\partial}{\partial \varphi}\right)^{k} \tilde{a}(\theta, \varphi)\right) f(\varphi) d \varphi \quad \text { or } \quad(A f)(\theta)=\int_{0}^{2 \pi}\left(\left(\frac{\partial}{\partial \theta}\right)^{k} \tilde{a}(\theta, \varphi)\right) f(\varphi) d \varphi
$$

Then we have $\|A f\|_{s} \leq C\|a\|_{s+k}\|f\|_{1 / 2+\varepsilon}$.

Proof. By (4.5), the former integral operator can be rewritten in the form

$$
(A f)(\theta)=(-1)^{k} \pi i \mathrm{e}^{-i \theta}\left\{[a, i \operatorname{sgn} D](i D)^{k} f(\theta)-i a(\theta) P_{0}(i D)^{k} f+i P_{0} a(i D)^{k} f\right\} .
$$

Since $P_{0} a(i D)^{k} f=P_{0}\left((i D)^{k} a\right) f$, the required estimate follows from Lemma 3.3 and the trivial estimate $\left\|P_{0} u v\right\|_{s}=\left\|P_{0} u v\right\|_{0} \leq\|u\|_{0}\|v\|_{0}$. For the latter one, the evaluation can be reduced to the former case, because we have

$$
\frac{\partial}{\partial \theta} \tilde{a}(\theta, \varphi)=-\frac{\partial}{\partial \varphi} \tilde{a}(\theta, \varphi)+\widetilde{a}^{\prime}(\theta, \varphi)-i \tilde{a}(\theta, \varphi) .
$$


The proof is complete.

Lemma 5.2. Let $k$ be a non-negative integer, $1 \leq p \leq \infty, 0<r<1$ and $\varepsilon>0$. Then we have

$$
\sup _{\theta}\left(\int_{0}^{2 \pi}\left|\left(\frac{\partial}{\partial \theta}\right)^{k} \tilde{a}(\theta, \varphi)\right|^{p} d \varphi\right)^{1 / p} \leq C\|a\|_{k+3 / 2-1 / p+\varepsilon}
$$

$$
\begin{gathered}
\left(\int_{0}^{2 \pi} \sup _{\theta_{1}}\left(\int_{0}^{2 \pi}\left|\left(\frac{\partial}{\partial \theta_{1}}\right)^{k}\left(\tilde{a}\left(\theta_{1}+\theta_{2}, \varphi\right)-\tilde{a}\left(\theta_{1}, \varphi\right)\right)\right|^{p} d \varphi\right)^{2 / p}\left|\mathrm{e}^{i \theta_{2}}-1\right|^{-(1+2 r)} d \theta_{2}\right)^{1 / 2} \\
\leq C\|a\|_{k+r+3 / 2-1 / p+\varepsilon}
\end{gathered}
$$

Here, the integrals with respect to $\varphi$ should be replaced by the essential supremum in the case $p=\infty$.

Proof. By the Fourier expansion of $a$ and Minkowski's inequality, we have

$$
\left(\int_{0}^{2 \pi}\left|\left(\frac{\partial}{\partial \theta}\right)^{\breve{k}} \tilde{a}(\theta, \varphi)\right|^{p} d \varphi\right)^{1 / p} \leq \sum_{n=-\infty}^{\infty}\left|a_{n}\right|\left(\int_{0}^{2 \pi}\left|\left(\frac{\partial}{\partial \theta}\right)^{k} \frac{\mathrm{e}^{i n \theta}-\mathrm{e}^{i n \varphi}}{\mathrm{e}^{i \theta}-\mathrm{e}^{i \varphi}}\right|^{p} d \varphi\right)^{1 / p}
$$

Since

$$
\frac{\mathrm{e}^{i n \theta}-\mathrm{e}^{i n \varphi}}{\mathrm{e}^{i \theta}-\mathrm{e}^{i \varphi}}= \begin{cases}\sum_{j=0}^{n-1} \mathrm{e}^{i(n-1-j) \theta+i j \varphi} & \text { for } n \geq 1, \\ 0 & \text { for } n=0, \\ \sum_{j=n}^{-1} \mathrm{e}^{i(n-1-j) \theta+i j \varphi} & \text { for } n \leq-1,\end{cases}
$$

it holds for $n \geq 1$ that

$$
\left|\left(\frac{\partial}{\partial \theta}\right)^{k} \frac{\mathrm{e}^{i n \theta}-\mathrm{e}^{i n \varphi}}{\mathrm{e}^{i \theta}-\mathrm{e}^{i \varphi}}\right|=\left|\sum_{j=0}^{n-1}(n-1-j)^{k} \mathrm{e}^{i j \varphi}\right| .
$$

Here, we see that

$$
\sum_{j=0}^{n-1}(n-1-j)^{k} \mathrm{e}^{i j \varphi}=\sum_{j=1}^{n-1}\left((n-j)^{k}-(n-j-1)^{k}\right) \frac{\mathrm{e}^{i j \varphi}-1}{\mathrm{e}^{i \varphi}-1}+\delta_{k 0} \mathrm{e}^{i(n-1) \varphi},
$$


where $\delta_{k 0}$ is Kronecker's symbol. Therefore

$$
\begin{aligned}
& \left(\int_{0}^{2 \pi}\left|\left(\frac{\partial}{\partial \theta}\right)^{k} \frac{\mathrm{e}^{i n \theta}-\mathrm{e}^{i n \varphi}}{\mathrm{e}^{i \theta}-\mathrm{e}^{i \varphi}}\right|^{p} d \varphi\right)^{1 / p} \\
& \leq \sum_{j=1}^{n-1}\left((n-j)^{k}-(n-j-1)^{k}\right)\left(\int_{0}^{2 \pi}\left|\frac{\mathrm{e}^{i j \varphi}-1}{\mathrm{e}^{i \varphi}-1}\right|^{p} d \varphi\right)^{1 / p}+\delta_{k 0}(2 \pi)^{1 / p} .
\end{aligned}
$$

In the same way as in the proof of Lemma 3.1, we obtain

$$
\begin{aligned}
& \left(\int_{0}^{2 \pi}\left|\frac{\mathrm{e}^{i j \varphi}-1}{\mathrm{e}^{i \varphi}-1}\right|^{p} d \varphi\right)^{1 / p} \\
& \leq \begin{cases}\left(\frac{4}{1-1 / p}\right)^{1 / p}\left(\frac{\pi}{2}\right)^{1+1 / p} j^{1-1 / p} & \text { if } p>1, \\
\pi^{2}(1+\log j) & \text { if } p=1\end{cases}
\end{aligned}
$$

for $j \geq 1$. On the other hand, it is clear that

$$
\sum_{j=1}^{n-1}\left((n-j)^{k}-(n-j-1)^{k}\right) \leq k 2^{k}(n-1)^{k}
$$

Hence we get

$$
\sup _{\theta}\left(\int_{0}^{2 \pi}\left|\left(\frac{\partial}{\partial \theta}\right)^{k} \frac{\mathrm{e}^{i n \theta}-\mathrm{e}^{i n \varphi}}{\mathrm{e}^{i \theta}-\mathrm{e}^{i \varphi}}\right|^{p} d \varphi\right)^{1 / p} \leq \begin{cases}C|n|^{k+1-1 / p} & \text { if } p>1 \\ C|n|^{k}(1+\log (1+|n|)) & \text { if } p=1\end{cases}
$$

for $n \geq 1$. We can show that the above estimate is also valid for $n \leq 0$. This fact and (5.5) lead us to (5.3).

As for (5.4), we have

$$
\begin{aligned}
& \left(\int_{0}^{2 \pi}\left|\left(\frac{\partial}{\partial \theta_{1}}\right)^{k}\left(\tilde{a}\left(\theta_{1}+\theta_{2}, \varphi\right)-\tilde{a}\left(\theta_{1}, \varphi\right)\right)\right|^{p} d \varphi\right)^{1 / p} \\
& \leq \sum_{n=-\infty}^{\infty}\left|a_{n}\right|\left(\int_{0}^{2 \pi}\left|\left(\frac{\partial}{\partial \theta_{1}}\right)^{k}\left(\frac{\mathrm{e}^{i n\left(\theta_{1}+\theta_{2}\right)}-\mathrm{e}^{i n \varphi}}{\mathrm{e}^{i\left(\theta_{1}+\theta_{2}\right)}-\mathrm{e}^{i \varphi}}-\frac{\mathrm{e}^{i n \theta_{1}}-\mathrm{e}^{i n \varphi}}{\mathrm{e}^{i \theta_{1}}-\mathrm{e}^{i \varphi}}\right)\right| d \varphi\right)^{p} \\
& =: \sum_{n=-\infty}^{\infty}\left|a_{n}\right| I_{n}\left(\theta_{1}, \theta_{2}\right) .
\end{aligned}
$$

We consider the case that $n \geq 1$ and $p>1$. By (5.6), we have 


$$
I_{n}\left(\theta_{1}, \theta_{2}\right)=\left(\int_{0}^{2 \pi}\left|\sum_{j=0}^{n-1}\left(\mathrm{e}^{i(n-1-j) \theta_{2}}-1\right)(n-1-j)^{k} \mathrm{e}^{i j \varphi}\right|^{p} d \varphi\right)^{1 / p}
$$

Here, it holds that

$$
\begin{aligned}
& \sum_{j=0}^{n-1}\left(\mathrm{e}^{i(n-1-j) \theta_{2}}-1\right)(n-1-j)^{k} \mathrm{e}^{i j \varphi} \\
& =\sum_{j=1}^{n-1}\left\{\left(\mathrm{e}^{i(n-j) \theta_{2}}-1\right)\left((n-j)^{k}-(n-j-1)^{k}\right)\right. \\
& \left.\quad+\mathrm{e}^{i(n-j-1) \theta_{2}}\left(\mathrm{e}^{i \theta_{2}}-1\right)(n-j-1)^{k}\right\} \frac{\mathrm{e}^{i j \varphi}-1}{\mathrm{e}^{i \varphi}-1} .
\end{aligned}
$$

This together with (5.7) shows that

$$
I_{n}\left(\theta_{1}, \theta_{2}\right) \leq C \sum_{j=1}^{n-1} j^{1-1 / p}\left\{\left|\mathrm{e}^{i(n-j) \theta_{2}}-1\right|\left((n-j)^{k}-(n-j-1)^{k}\right)+\left|\mathrm{e}^{i \theta_{2}}-1\right|(n-j-1)^{k}\right\} .
$$

On the other hand, by (5.8) it is clear that $I_{n}\left(\theta_{1}, \theta_{2}\right) \leq C|n|^{k+1-1 / p}$. Thus, using the similar calculation in the proof of Lemma 3.1, we see that

$$
\begin{aligned}
& \left(\int_{0}^{2 \pi}\left(\sup _{\theta_{1}} I_{n}\left(\theta_{1}, \theta_{2}\right)\right)^{2}\left|\mathrm{e}^{i \theta_{2}}-1\right|^{-(1+2 r)} d \theta_{2}\right)^{1 / 2} \\
& \leq C|n|^{k+1-1 / p}\left(\int_{\pi / n}^{2 \pi-\pi / n}\left|\mathrm{e}^{i \theta_{2}}-1\right|^{-(1+2 r)} d \theta_{2}\right)^{1 / 2} \\
& \quad+C \sum_{j=1}^{n-1} j^{1-1 / p}\left\{\left((n-j)^{k}-(n-j-1)^{k}\right)\left(\int_{0}^{2 \pi} \frac{\left|\mathrm{e}^{i(n-j) \theta_{2}}-1\right|^{2}}{\left|\mathrm{e}^{i \theta_{2}}-1\right|^{1+2 r}} d \theta_{2}\right)^{1 / 2}\right. \\
& \left.\quad+(n-j-1)^{k}\left(\int_{0}^{\pi / n}+\int_{2 \pi-\pi / n}^{2 \pi}\left|\mathrm{e}^{i \theta_{2}}-1\right|^{1-2 r} d \theta_{2}\right)^{1 / 2}\right\} \\
& \leq C|n|^{k+r+1-1 / p} .
\end{aligned}
$$

Similarly, we can show that the above estimate is also valid for $n \leq 0$. This fact and (5.9) show that (5.4) holds for $p>1$. The proof in the case $p=1$ is almost the same. The proof is complete.

Lemma 5.3. Let $k$ be a non-negative integer, $2 \leq q<\infty$ and $0<r<1$. Then we have 


$$
\left(\int_{0}^{2 \pi}\left(\int_{0}^{2 \pi}\left|\left(\frac{\partial}{\partial \theta}\right)^{k} \tilde{a}(\theta, \varphi)\right|^{q} d \varphi\right)^{2 / q} d \theta\right)^{1 / 2} \leq C\|a\|_{k+1-1 / q}
$$

$$
\begin{gathered}
\left(\int_{0}^{2 \pi} \int_{0}^{2 \pi}\left(\int_{0}^{2 \pi}\left|\left(\frac{\partial}{\partial \theta_{1}}\right)^{k}\left(\tilde{a}\left(\theta_{1}+\theta_{2}, \varphi\right)-\tilde{a}\left(\theta_{1}, \varphi\right)\right)\right|^{q} d \varphi\right)^{2 / q} d \theta_{1}\left|\mathrm{e}^{i \theta_{2}}-1\right|^{-(1+2 r)} d \theta_{2}\right)^{1 / 2} \\
\leq C\|a\|_{k+r+1-1 / q}
\end{gathered}
$$

Proof. By the Fourier expansion of $a$ and (5.6), we have

$$
\begin{aligned}
\left(\frac{\partial}{\partial \theta}\right)^{k} \tilde{a}(\theta, \varphi)= & \sum_{j=0}^{\infty}\left(\sum_{n=j+1}^{\infty} a_{n}(i(n-1-j))^{k} \mathrm{e}^{i(n-1-j) \theta}\right) \mathrm{e}^{i j \varphi} \\
& +\sum_{j=-\infty}^{-1}\left(\sum_{n=-\infty}^{j} a_{n}(i(n-1-j))^{k} \mathrm{e}^{i(n-1-j) \theta}\right) \mathrm{e}^{i j \varphi} .
\end{aligned}
$$

This and the Sobolev imbedding $H^{1 / 2-1 / q} \hookrightarrow L^{q}$ imply that

$$
\begin{aligned}
& \left(\left.\int_{0}^{2 \pi}\left|\left(\frac{\partial}{\partial \theta}\right)^{k} \tilde{a}(\theta, \varphi)\right| q\right|^{q} d \varphi\right)^{2 / q} \\
& \leq C \sum_{j=0}^{\infty}(1+|j|)^{2(1 / 2-1 / q)} \mid \sum_{n=j+1}^{\infty}\left(\left.a_{n}(i(n-1-j))^{k} \mathrm{e}^{i(n-1-j) \theta}\right|^{2}\right. \\
& \quad+C \sum_{j=-\infty}^{-1}(1+|j|)^{2(1 / 2-1 / q)}\left|\sum_{n=-\infty}^{j} a_{n}(i(n-1-j))^{k} \mathrm{e}^{i(n-1-j) \theta}\right|^{2} .
\end{aligned}
$$

Integrating this with respect to $\theta$ and using Parseval's formula, we obtain

$$
\begin{aligned}
& \int_{0}^{2 \pi}\left(\int_{0}^{2 \pi}\left|\left(\frac{\partial}{\partial \theta}\right)^{k} \tilde{a}(\theta, \varphi)\right| q \varphi\right)^{2 / q} d \theta \\
& \quad \leq C\left(\sum_{n=1}^{\infty} \sum_{j=0}^{n-1}+\sum_{n=-\infty}^{-1} \sum_{j=n}^{-1}\right)(1+|j|)^{2(1 / 2-1 / q)}|n-1-j|^{2 k}\left|a_{n}\right|^{2} \\
& \quad \leq C \sum_{n=-\infty}^{\infty}|n|^{2(k+1-1 / q)}\left|a_{n}\right|^{2} \leq C\|a\|_{k+1-1 / q}^{2}
\end{aligned}
$$

By virtue of Lemma 3.1, we can show (5.11) in the same way as above. The proof is finished.

Lemma 5.4. Let $j$ and $k$ be non-negative integers, $0<s \leq 1 / 2, \varepsilon>0$ and 


$$
I(\theta)=\int_{0}^{2 \pi}\left|\left(\frac{\partial}{\partial \theta}\right)^{j} \tilde{a}(\theta, \varphi)\right|\left|\left(\frac{\partial}{\partial \theta}\right)^{k} \tilde{b}(\theta, \varphi)\right| d \varphi
$$

Then we have $\|I\|_{0} \leq C\|a\|_{j+1 / 2+s+\varepsilon}\|b\|_{k+1-s}$.

Proof. Put $p=1 /(1-s)$ and $q=1 / s$. Then it holds that $1<p \leq 2 \leq q<\infty$ and $1 / p+1 / q=1$. Hence, by Hölder's inequality, (5.3) and (5.10), we obtain

$$
\begin{aligned}
\|I\|_{0}^{2} & \leq \sup _{\theta}\left(\left.\int_{0}^{2 \pi}\left|\left(\frac{\partial}{\partial \theta}\right)^{j} \tilde{a}(\theta, \varphi)\right|\right|^{p} d \varphi\right)^{2 / p} \int_{0}^{2 \pi}\left(\int_{0}^{2 \pi}\left|\left(\frac{\partial}{\partial \theta}\right)^{k} \tilde{b}(\theta, \varphi)\right|^{q} d \varphi\right)^{2 / q} d \theta \\
& \leq C\|a\|_{j+3 / 2-1 / p+\varepsilon}^{2}\|b\|_{k+1-1 / q}^{2} .
\end{aligned}
$$

This shows the required estimate. The proof is complete.

Lemma 5.5. Let $j$ and $k$ be non-negative integers, $0<s \leq 1 / 2,0<r<1, \varepsilon>$ 0 and

$$
I\left(\theta_{1}, \theta_{2}\right)=\int_{0}^{2 \pi}\left|\left(\frac{\partial}{\partial \theta_{1}}\right)^{j}\left(\tilde{a}\left(\theta_{1}+\theta_{2}, \varphi\right)-\tilde{a}\left(\theta_{1}, \varphi\right)\right)\right|\left|\left(\frac{\partial}{\partial \theta_{1}}\right)^{k} \tilde{b}\left(\theta_{1}, \varphi\right)\right| d \varphi .
$$

Then we have

$$
\left(\int_{0}^{2 \pi} \int_{0}^{2 \pi} \frac{\left|I\left(\theta_{1}, \theta_{2}\right)\right|^{2}}{\left|\mathrm{e}^{i \theta_{2}}-1\right|^{1+2 r}} d \theta_{1} d \theta_{2}\right)^{1 / 2} \leq C\|a\|_{j+r+1 / 2+s-\varepsilon}\|b\|_{k+1-s} .
$$

Proof. By virtue of (5.4) and (5.11), the above estimate can be proved in the same way as the previous lemma. The proof is finished.

Lemma 5.6. Let $k$ be a non-negative integer, $0 \leq r<1, \varepsilon>0$ and $A$ the integral operator of the form

$$
(A f)(\theta)=\int_{0}^{2 \pi}\left(\left(\frac{\partial}{\partial \theta}\right)^{k} \tilde{a}(\theta, \varphi)\right) F(\tilde{u}(\theta, \varphi)) f(\varphi) d \varphi,
$$

where $F$ and $u$ are of the same as in (5.1). Then we have $\|A f\|_{r} \leq$ $C\|a\|_{k+r}\left(1+\|u\|_{2+r}\right)^{2}\|f\|_{1 / 2+\varepsilon}$.

Proof. To begin with, we consider the case $r=0$. 


$$
\begin{aligned}
(A f)(\theta)= & F\left(\left(i \mathrm{e}^{i \theta}\right)^{-1} u^{\prime}(\theta)\right) \int_{0}^{2 \pi}\left(\left(\frac{\partial}{\partial \theta}\right)^{k} \tilde{a}(\theta, \varphi)\right) f(\varphi) d \varphi \\
& +\int_{0}^{2 \pi}\left(\left(\frac{\partial}{\partial \theta}\right)^{k} \tilde{a}(\theta, \varphi)\right)\left\{F(\tilde{u}(\theta, \varphi))-F\left(\left(\mathrm{ie}^{i \theta}\right)^{-1} u^{\prime}(\theta)\right)\right\} f(\varphi) d \varphi \\
= & :\left(A_{1} f\right)(\theta)+\left(A_{2} f\right)(\theta) .
\end{aligned}
$$

It follows from Lemma 5.1 that $\left\|A_{1} f\right\|_{0} \leq C\|a\|_{k}\|f\|_{1 / 2+\varepsilon}$. Since

$$
\tilde{u}(\theta, \varphi)-\left(i \mathrm{e}^{i \theta}\right)^{-1} u^{\prime}(\theta)=i \mathrm{e}^{-i \theta}\left(\mathrm{e}^{i \theta}-\mathrm{e}^{i \varphi}\right) \frac{\partial}{\partial \theta} \tilde{u}(\theta, \varphi)
$$

we can rewrite $A_{2}$ as

$$
\left(A_{2} f\right)(\theta)=\sum_{j=1}^{N} \int_{0}^{2 \pi}\left(\left(\frac{\partial}{\partial \theta}\right)^{k} \tilde{a}(\theta, \varphi)\right) F_{j}(\theta, \varphi) i \mathrm{e}^{-i \theta}\left(\mathrm{e}^{i \theta}-\mathrm{e}^{i \varphi}\right) \frac{\partial}{\partial \theta} \tilde{u}_{j}(\theta, \varphi) f(\varphi) d \varphi
$$

where

$$
F_{j}(\theta, \varphi)=\int_{0}^{1} \frac{\partial F}{\partial z_{j}}\left(t \tilde{u}(\theta, \varphi)+(1-t)\left(i \mathrm{e}^{i \theta}\right)^{-1} u^{\prime}(\theta)\right) d t, \quad j=1, \ldots, N
$$

For $k=0$, we have

$$
\begin{aligned}
\left(A_{2} f\right)(\theta)= & \sum_{j=1}^{N} i \mathrm{e}^{-i \theta} a(\theta) \int_{0}^{2 \pi} F_{j}(\theta, \varphi) \frac{\partial}{\partial \theta} \tilde{u}_{j}(\theta, \varphi) f(\varphi) d \varphi \\
& -\sum_{j=1}^{N} i \mathrm{e}^{-i \theta} \int_{0}^{2 \pi} F_{j}(\theta, \varphi) \frac{\partial}{\partial \theta} \tilde{u}_{j}(\theta, \varphi) a(\varphi) f(\varphi) d \varphi .
\end{aligned}
$$

Using (5.3) with $k=p=1$ and $\varepsilon=1 / 2$, well-known estimate $|f(\varphi)| \leq C\|f\|_{1 / 2+\varepsilon}$ and Schwarz's inequality, we get

$$
\left|\left(A_{2} f\right)(\theta)\right| \leq C|a(\theta)|\|u\|_{2}\|f\|_{1 / 2+\varepsilon}+C\left(\int_{0}^{2 \pi}\left|\frac{\partial}{\partial \theta} \tilde{u}(\theta, \varphi)\right|^{2} d \varphi\right)^{1 / 2}\|a\|_{0}\|f\|_{1 / 2+\varepsilon} .
$$

This together with (5.10) with $k=1$ and $q=2$ implies that $\left\|A_{2} f\right\|_{0}$ $\leq C\|a\|_{0}\|u\|_{2}\|f\|_{1 / 2+\varepsilon}$. For $k \geq 1$, we differentiate the identity $a(\theta)-a(\varphi)$ $=\tilde{a}(\theta, \varphi)\left(\mathrm{e}^{i \theta}-\mathrm{e}^{i \varphi}\right) k$-times with respect to $\theta$ and get

$$
\left(\frac{\partial}{\partial \theta}\right)^{k} \tilde{a}(\theta, \varphi)=\frac{1}{\mathrm{e}^{i \theta}-\mathrm{e}^{i \varphi}}\left\{a^{(k)}(\theta)-\mathrm{e}^{i \theta} \sum_{l=0}^{k-1} i^{k-l}\left(\begin{array}{l}
k \\
l
\end{array}\right)\left(\frac{\partial}{\partial \theta}\right)^{l} \tilde{a}(\theta, \varphi)\right\},
$$


where $a^{(k)}(\theta)=(d / d \theta)^{k} a(\theta)$. Therefore, it holds that

$$
\begin{aligned}
\left(A_{2} f\right)(\theta)= & \sum_{j=1}^{N} i \mathrm{e}^{-i \theta} a^{(k)}(\theta) \int_{0}^{2 \pi} F_{j}(\theta, \varphi) \frac{\partial}{\partial \theta} \tilde{u}_{j}(\theta, \varphi) f(\varphi) d \varphi \\
& -\sum_{j=1}^{N} \sum_{l=0}^{k-1} i^{k-l+1}\left(\begin{array}{l}
k \\
l
\end{array}\right) \int_{0}^{2 \pi} F_{j}(\theta, \varphi)\left(\left(\frac{\partial}{\partial \theta}\right)^{l} \tilde{a}(\theta, \varphi)\right)\left(\frac{\partial}{\partial \theta} \tilde{u}_{j}(\theta, \varphi)\right) f(\varphi) d \varphi .
\end{aligned}
$$

By the same evaluation as above and Lemma 5.4 with $j=l, k=1$ and $s=\varepsilon=1 / 4$, we obtain $\left\|A_{2} f\right\|_{0} \leq C\|a\|_{k}\|u\|_{2}\|f\|_{1 / 2+\varepsilon}$. Hence, the required estimate is proved in the case $r=0$. Furthermore, we can show it for $0<r<1$ in the same way as above, because we have Lemmas 3.1, 5.5 and (5.4). The proof is finished.

Lemma 5.7. Let $k$ be a non-negative integer, $0 \leq r<1, \varepsilon>0$ and $A$ the integral operator of the form

$$
(A f)(\theta)=\int_{0}^{2 \pi}\left(\left(\frac{\partial}{\partial \theta}\right)^{k} \tilde{a}(\theta, \varphi)\right)\left(\prod_{j=1}^{M} \tilde{b}_{j}(\theta, \varphi)\right) F(\tilde{u}(\theta, \varphi)) f(\varphi) d \varphi
$$

where $F$ and $u$ are of the same as in (5.1). Then we have

$$
\|A f\|_{r} \leq C\|a\|_{k+r}\left\|b_{1}\right\|_{2+r} \cdots\left\|b_{M}\right\|_{2+r}\left(1+\|u\|_{2+r}\right)^{2}\|f\|_{1 / 2+\varepsilon} .
$$

Proof. This integral operator is the same type as in Lemma 5.6 with $F(z)$ replaced by $z_{1} \cdots z_{M} F\left(z_{M+1}, \ldots, z_{M+N}\right)$. By noting this fact and that $A$ depends linearly on each $b_{j}, j=1, \ldots, M$, the required estimate follows from Lemma 5.6. The proof is complete.

Lemma 5.8. Let $s \geq 2, \varepsilon>0$ and $A$ be the integral operator defined by (5.1) with $M=1$. Then we have $\|A f\|_{s} \leq C\left\|a_{1}\right\|_{s}\left(1+\|u\|_{s}\right)^{[s]+1}\|f\|_{1 / 2+\varepsilon}$, where $[s]$ is the integer part of $s$.

Proof. Put $m=[s]$ and $r=s-[s]$. Then, it holds that $m \geq 2,0 \leq r<1$ and $s=m+r$. By Lemma 5.6, we have $\|A f\|_{r} \leq C\left\|a_{1}\right\|_{r}\left(1+\|u\|_{2+r}\right)^{2}\|f\|_{1 / 2+\varepsilon}$. Therefore, it is sufficient to evaluate $\left\|(d / d \theta)^{m} A f\right\|_{r}$.

$$
\left(\frac{d}{d \theta}\right)^{m}(A f)(\theta)=\int_{0}^{2 \pi}\left(\left(\frac{\partial}{\partial \theta}\right)^{m} \tilde{a}_{1}(\theta, \varphi)\right) F(\tilde{u}(\theta, \varphi)) f(\varphi) d \varphi
$$




$$
\begin{aligned}
& +\sum_{j=1}^{M} \int_{0}^{2 \pi}\left(\left(\frac{\partial}{\partial \theta}\right)^{m} \tilde{u}_{j}(\theta, \varphi)\right) \tilde{a}_{1}(\theta, \varphi) \frac{\partial F}{\partial z_{j}}(\tilde{u}(\theta, \varphi)) f(\varphi) d \varphi \\
& +\sum C \int_{0}^{2 \pi}\left(\left(\frac{\partial}{\partial \theta}\right)^{\sigma} \tilde{a}_{1}(\theta, \varphi)\right) \frac{\partial^{\alpha} F}{\partial z^{\alpha}}(\tilde{u}(\theta, \varphi)) \prod_{k=1}^{\lambda}\left(\left(\frac{\partial}{\partial \theta}\right)^{k} \tilde{u}(\theta, \varphi)\right)^{\mu_{k}} f(\varphi) d \varphi \\
= & :\left(A_{1} f\right)(\theta)+\left(A_{2} f\right)(\theta)+\left(A_{3} f\right)(\theta),
\end{aligned}
$$

where the symbol $\Sigma$ in $A_{3} f$ means the summation over all $(\sigma, \alpha, \lambda, \mu)$ satisfying

$$
\left\{\begin{array}{l}
0 \leq \sigma \leq m-1, \quad 1 \leq|\alpha| \leq m, \quad 1 \leq \lambda \leq m-1, \\
\sigma+\sum_{k=1}^{\lambda} k\left|\mu_{k}\right|=m, \quad \sum_{k=1}^{\lambda}\left|\mu_{k}\right|=|\alpha| .
\end{array}\right.
$$

From Lemmas 5.6 and 5.7, it follows that $\left\|A_{1} f\right\|_{r} \leq C\left\|a_{1}\right\|_{m+r}\left(1+\|u\|_{2+r}\right)\|f\|_{1 / 2+\varepsilon}$ and that $\left\|A_{2} f\right\|_{r} \leq C\|u\|_{m+r}\left\|a_{1}\right\|_{2+r}\left(1+\|u\|_{2+r}\right)\|f\|_{1 / 2+\varepsilon}$, respectively. Noting that

$$
\sup _{\theta, \varphi}\left|\left(\frac{\partial}{\partial \theta}\right)^{k} \tilde{u}(\theta, \varphi)\right| \leq C\|u\|_{k+2}
$$

which comes from (5.3) with $p=\infty$ and $\varepsilon=1 / 2$, we proceed to evaluate

$$
I:=\left(\left(\frac{\partial}{\partial \theta}\right)^{\sigma} \tilde{a}_{1}(\theta, \varphi)\right) \frac{\partial^{\alpha} F}{\partial z^{\alpha}}(\tilde{u}(\theta, \varphi)) \prod_{k=1}^{\lambda}\left(\left(\frac{\partial}{\partial \theta}\right)^{k} \tilde{u}(\theta, \varphi)\right)^{\mu_{k}}
$$

In the case $\lambda \leq m-2$, we have

$$
|I| \leq C\left|\left(\frac{\partial}{\partial \theta}\right)^{\sigma} \tilde{a}_{1}(\theta, \varphi)\right|\|u\|_{m}^{|\alpha|} .
$$

In the case $\lambda=m-1$, if $m \geq 3$, then $\left|\mu_{\lambda}\right|=1$ and

$$
|I| \leq C\left|\left(\frac{\partial}{\partial \theta}\right)^{\sigma} \tilde{a}_{1}(\theta, \varphi)\left\|\left(\frac{\partial}{\partial \theta}\right)^{\lambda} \tilde{u}(\theta, \varphi) \mid\right\| u \|_{m}^{|\alpha|-1} .\right.
$$

If $m=2$ and $\sigma=\left|\mu_{\lambda}\right|=1$, then the above estimate is still valid. If $m=2, \sigma=0$ and $\left|\mu_{\lambda}\right|=2$, then

$$
|I| \leq C\left\|a_{1}\right\|_{2}\left|\frac{\partial}{\partial \theta} u(\theta, \varphi)\right|^{2}
$$


By these estimates, (5.10) with $q=2$ and Lemma 5.4 with $s=\varepsilon=1 / 4$, we obtain $\left\|A_{3} f\right\|_{0} \leq C\left\|a_{1}\right\|_{m}\left(1+\|u\|_{m}\right)^{m}\|f\|_{1 / 2+\varepsilon}$. Moreover, we can show that $\left\|A_{3} f\right\|_{r}$ $\leq C\left\|a_{1}\right\|_{m+r}\left(1+\|u\|_{m+r}\right)^{m+1}\|f\|_{1 / 2+\varepsilon}$ in the same way as above, because we have (5.4), (5.11), Lemmas 3.1 and 5.5. The proof is complete.

Lemma 5.9. Let $s \geq 2, \varepsilon>0$ and $A=A(a, u)$ be the integral operator defined by (5.1). Then we have

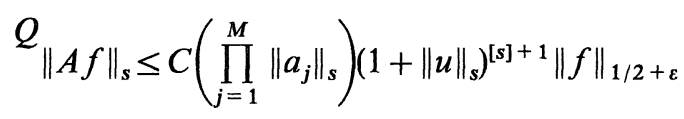

and

$$
\begin{aligned}
& \left\|A\left(a^{1}, u^{1}\right) f-A\left(a^{2}, u^{2}\right) f\right\|_{s} \\
& \leq C\left(1+\left\|a^{1}\right\|_{s}+\left\|a^{2}\right\|_{s}\right)^{M}\left(1+\left\|u^{1}\right\|_{s}+\left\|u^{2}\right\|_{s}\right)^{[s]+1}\left(\left\|a^{1}-a^{2}\right\|_{s}+\left\|u^{1}-u^{2}\right\|_{s}\right)\|f\|_{1 / 2+\varepsilon} .
\end{aligned}
$$

Proof. By Lemma 5.8 and the arguments in the proof of Lemma 5.7, we obtain the first estimate. In order to show the second one, we write

$$
A\left(a^{1}, u^{1}\right) f-A\left(a^{2}, u^{2}\right) f=\int_{0}^{1} B(t) f d t
$$

where $B(t)$ is almost the same type as $A$ and $\|B(t) f\|_{s}$ can be estimated by the right hand side of the required second inequality uniformly in $t \in[0,1]$, because of Lemma 5.8. The proof is complete.

Next, we consider the integral operator defined by (5.2). The estimation for it is straightforward and easier than the previous one. This is the reason why we state the following lemma without proof.

Lemma 5.10. Let $s \geq 1$ and $A=A(a, b, u, v)$ be the integral operator defined by (5.2). Then we have

$$
\|A f\|_{s} \leq C\left(\prod_{j=1}^{M}\left(\left\|a_{j}\right\|_{s}+\left\|b_{j}\right\|_{1}\right)\right)\left(1+\|u\|_{s}+\|v\|_{1}\right)^{[s]+1}\|f\|_{0}
$$

and

$$
\begin{aligned}
& \left\|A\left(a^{1}, b^{1}, u^{1}, v^{1}\right) f-A\left(a^{2}, b^{2}, u^{2}, v^{2}\right) f\right\|_{s} \\
& \quad \leq C\left(1+\left\|a^{1}\right\|_{s}+\left\|a^{2}\right\|_{s}+\left\|b^{1}\right\|_{1}+\left\|b^{2}\right\|_{1}\right)^{M}
\end{aligned}
$$




$$
\begin{aligned}
& \times\left(1+\left\|u^{1}\right\|_{s}+\left\|u^{2}\right\|_{s}+\left\|v^{1}\right\|_{1}+\left\|v^{2}\right\|_{1}\right)^{[s]+1} \\
& \times\left(\left\|a^{1}-a^{2}\right\|_{s}+\left\|b^{1}-b^{2}\right\|_{1}+\left\|u^{1}-u^{2}\right\|_{s}+\left\|v^{1}-v^{2}\right\|_{1}\right)\|f\|_{0} .
\end{aligned}
$$

Now, we are ready to investigate the operator $K_{1}$ defined by (4.13).

Lemma 5.11. For the operator $K_{1}$ defined by (4.13), we have

$$
\left\{\begin{array}{lll}
K_{1}(X, b ; X, b) \in L_{0}(2, s ; 3) & \text { for } & s \geq 3, \\
K_{1}\left(X, b, b^{\prime} ; X, b, b^{\prime}\right) \in L_{0}(2, s ; 2) & \text { for } & s \geq 2 .
\end{array}\right.
$$

Moreover, it holds that $T_{\theta} K_{1}(X, b)=K_{1}\left(T_{\theta} X, T_{\theta} b\right) T_{\theta}$ for $\theta \in \mathbf{R}^{1}$.

Proof. By virtue of Lemmas 5.9 and 5.10, for the operators $A_{j}, j=1, \ldots, 22$, we have $A_{1}\left(X_{1} ; 0\right) \in L_{0}(1, s ; 0)$ for $s \geq 2$, etc., and $T_{\theta} A_{1}\left(X_{1}\right)=A_{1}\left(T_{\theta} X_{1}\right) T_{\theta}$ for $\theta \in \mathbf{R}^{1}$, etc. Therefore, for $j=3$ and 4 we obtain

$$
\left\{\begin{array}{lll}
B_{j}(X, b ; X, b) \in L_{0}(2, s ; 2) & \text { for } & s \geq 3, \\
B_{j}\left(X, b, b^{\prime} ; X, b, b^{\prime}\right) \in L_{0}(2, s ; 2) & \text { for } & s \geq 2
\end{array}\right.
$$

and $T_{\theta} B_{j}(X, b)=B_{j}\left(T_{\theta} X, T_{\theta} b\right) T_{\theta}$. These facts and Lemma 3.4 imply that

$$
M_{1}\left(X, b, b^{\prime} ; X, b, b^{\prime}\right)=\left(1-B_{3}\right)^{-1} \in L(2,2 ; 2) .
$$

Hence, for $j=1$ and 2 we get

$$
\left\{\begin{array}{lll}
B_{j}(X, b ; X, b) \in L_{0}(2, s ; 3) & \text { for } & s \geq 3, \\
B_{j}\left(X, b, b^{\prime} ; X, b, b^{\prime}\right) \in L_{0}(2, s ; 2) & \text { for } & s \geq 2
\end{array}\right.
$$

and $T_{\theta} B_{j}(X, b)=B_{j}\left(T_{\theta} X, T_{\theta} b\right) T_{\theta}$. Using these facts and Lemma 3.4 again, we see that

$$
M_{2}\left(X, b, b^{\prime} ; X, b, b^{\prime}\right)=\left(1-B_{1}\right)^{-1} \in L(2,2 ; 2) .
$$

Consequently, we obtain the desired results. The proof is complete.

We introduce the following notations which are the same as those in [7]. Assuming that $X$ depends also on $t$, we define

$$
K_{1, k, l}\left(X, \ldots, \partial_{t}^{k} \partial_{\theta}^{l} X, b, \ldots, \partial_{\theta}^{l} b ; X, b\right), \quad k, l=0,1,2, \ldots,
$$

inductively by 


$$
\begin{cases}K_{1,0.0}=K_{1}, \quad K_{1,0, l}=\left[\frac{\partial}{\partial \theta}, K_{1,0, l-1}\right], & l=1,2,3, \ldots \\ K_{1, k, l}=\left[\frac{\partial}{\partial t}, K_{1, k-1, l}\right], \quad k=1,2,3, \ldots, & l=0,1,2, \ldots\end{cases}
$$

and replace $\partial_{t}^{p} \partial_{\theta}^{q} X$ by $X^{p q}$. Since $[R, S T]=[R, S] T+S[R, T],\left[R,(1-T)^{-1}\right]$ $=(1-T)^{-1}[R, T](1-T)^{-1}$ and

$$
\begin{aligned}
& \left(\frac{\partial}{\partial \theta}+\frac{\partial}{\partial \varphi}\right) \frac{a(\theta)-a(\varphi)}{\mathrm{e}^{i \theta}-\mathrm{e}^{i \varphi}}=\frac{a^{\prime}(\theta)-a^{\prime}(\varphi)}{\mathrm{e}^{i \theta}-\mathrm{e}^{i \varphi}}-i \frac{a(\theta)-a(\varphi)}{\mathrm{e}^{i \theta}-\mathrm{e}^{i \varphi}}, \\
& \left(\frac{\partial}{\partial \theta}+\frac{\partial}{\partial \varphi}\right) \frac{a(\theta)+b(\varphi)}{\mathrm{e}^{i \theta}-\beta \mathrm{e}^{i \varphi}}=\frac{a^{\prime}(\theta)+b^{\prime}(\varphi)}{\mathrm{e}^{i \theta}-\beta \mathrm{e}^{i \varphi}}-i \frac{a(\theta)+b(\varphi)}{\mathrm{e}^{i \theta}-\beta \mathrm{e}^{i \varphi}},
\end{aligned}
$$

we see that the operators $K_{1, k . l}$ are the similar type as $K_{1}$. Therefore, we obtain the following lemma.

Lemma 5.12. For non-negative integers $k$ and $l$, we have

$$
\left\{\begin{array}{l}
K_{1 . k, l}\left(X^{00}, \ldots, X^{k l}, b, \ldots, \partial_{\theta}^{l} b ; X^{00}, b\right) \in L_{0}(2, s ; 3) \quad \text { for } s \geq 3, \\
K_{1 . k . l}\left(X^{00}, \ldots, X^{k l}, b, \ldots, \partial_{\theta}^{l+1} b ; X^{00}, b, b^{\prime}\right) \in L_{0}(2, s ; 2) \quad \text { for } s \geq 2
\end{array}\right.
$$

\section{§6. Quasi-linearization}

Assuming that $X$ satisfies (2.9) and (2.10), and putting

$$
Y=X_{t t}, \quad Z=X_{\theta}, \quad W=(X, Y, Z), \quad W^{\prime}=\left(X, Y_{1}\right),
$$

we derive a quasi-linear system of equations for $W$. It follows from (2.10) that

$$
\begin{gathered}
\partial_{t}^{k} X_{2 t}=K\left(1+X_{2}\right) \partial_{t}^{k} X_{1 t}+F_{k 0}\left(X, \ldots, \partial_{t}^{k} X\right), \\
\partial_{t}^{k} \partial_{\theta}^{l} X_{2 t}=K\left(1+X_{2}\right) \partial_{t}^{k} \partial_{\theta}^{l} X_{1 t}+F_{k l}\left(X, \ldots, \partial_{t}^{k} \partial_{\theta}^{l} X, \partial_{t}^{k+1} X_{1}\right),
\end{gathered}
$$

where $k=0,1,2, \ldots, l=1,2,3, \ldots$, and $F_{k l}=\left[\partial_{t}^{k} \partial_{\theta}^{l}, K\left(1+X_{2}\right)\right] X_{1 t}$. Although $F_{k l}$ depends also on $b$ and it's derivatives, we do not indicate them. Similar simplification of notations will be used in the following without any comment. By (6.2) with $k=2$, we have

$$
Y_{2 t}=K\left(1+X_{2}\right) Y_{1 t}+F_{20}\left(X, X_{t}, Y\right)=: f_{2}\left(W, W_{t}^{\prime}\right)
$$


Differentiating (2.9) with respect to $t$, we obtain

$$
\begin{aligned}
(1+ & \left.X_{2}\right)\left(\left(1+X_{2}\right) Y_{1}+2 X_{1 t} X_{2 t}\right) Z_{1 t}+\left(Y_{2}-\left(1+X_{2}\right) X_{1 t}^{2}+\kappa\left(1+X_{2}\right)^{-2}\right) Z_{2 t} \\
& +\left(1+Z_{1}\right)\left(\left(1+X_{2}\right)^{2} Y_{1 t}+4\left(1+X_{2}\right) X_{2 t} Y_{1}+2 X_{1 t} X_{2 t}^{2}+2\left(1+X_{2}\right) X_{1 t} Y_{2}\right) \\
& +Z_{2}\left(Y_{2 t}-X_{2 t} X_{1 t}^{2}-2\left(1+X_{2}\right) X_{1 t} Y_{1}-2 \kappa X_{2 t}\left(1+X_{2}\right)^{-3}\right)=0
\end{aligned}
$$

It follows from (6.3) with $k=0$ and $l=1$ that

$$
Z_{2 t}=-i \operatorname{sgn} D\left(1+X_{2}\right) Z_{1 t}+(i \operatorname{sgn} D+K)\left(1+X_{2}\right)(i D) X_{1 t}+F_{01}\left(X, Z, X_{1 t}\right) .
$$

Eliminating $Z_{2 t}$ from (6.5) and (6.6), and substituting $f_{2}$ for $Y_{2 t}$, we see that

$$
\begin{aligned}
Z_{1 t}=- & \left\{\left(1+X_{2}\right)^{2} Y_{1}+2\left(1+X_{2}\right) X_{1 t} X_{2 t}\right. \\
& \left.-\left(Y_{2}-\left(1+X_{2}\right) X_{1 t}^{2}+\kappa\left(1+X_{2}\right)^{-2}\right) i \operatorname{sgn} D\left(1+X_{2}\right)\right\}^{-1} \\
\times & \left\{\left(Y_{2}-\left(1+X_{2}\right) X_{1 t}^{2}+\kappa\left(1+X_{2}\right)^{-2}\right)\right. \\
& \times\left((i \operatorname{sgn} D+K)\left(1+X_{2}\right)(i D) X_{1 t}+F_{01}\left(X, Z, X_{1 t}\right)\right) \\
& +Z_{2}\left(f_{2}\left(W, W_{t}^{\prime}\right)-X_{2 t} X_{1 t}^{2}-2\left(1+X_{2}\right) X_{1 t} Y_{1}-2 \kappa X_{2 t}\left(1+X_{2}\right)^{-3}\right) \\
& \left.+\left(1+Z_{1}\right)\left(\left(1+X_{2}\right)^{2} Y_{1 t}+4\left(1+X_{2}\right) X_{2 t} Y_{1}+2 X_{1 t} X_{2 t}^{2}+2\left(1+X_{2}\right) X_{1 t} Y_{2}\right)\right\} \\
=: & f_{3}\left(W, W_{t}^{\prime}\right) .
\end{aligned}
$$

This and (6.6) imply that

$$
\begin{aligned}
Z_{2 t} & =-i \operatorname{sgn} D\left(1+X_{2}\right) f_{3}\left(W, W_{t}^{\prime}\right)+(i \operatorname{sgn} D+K)\left(1+X_{2}\right)(i D) X_{1 t}+F_{01}\left(X, Z, X_{1 t}\right) \\
& =: f_{4}\left(W, W_{t}^{\prime}\right) .
\end{aligned}
$$

By virtue of (6.2) with $k=3$ and (6.3) with $k=l=1$, it holds that

$$
\left\{\begin{array}{l}
Y_{2 t t}=K\left(1+X_{2}\right) Y_{1 t t}+F_{30}\left(X, X_{t}, Y, Y_{t}\right), \\
Y_{2 \theta}=K\left(1+X_{2}\right) Y_{1 \theta}+F_{11}\left(X, X_{t}, Z, Z_{t}, Y_{1}\right) .
\end{array}\right.
$$

Differentiating (2.9) two times with respect to $t$ and putting these into the result, we obtain

$$
\begin{aligned}
((1+ & \left.\left.Z_{1}\right)\left(1+X_{2}\right)^{2}+Z_{2} K\left(1+X_{2}\right)\right) Y_{1 t t} \\
+ & \left\{\left(1+X_{2}\right)^{2} Y_{1}+2\left(1+X_{2}\right) X_{1 t} X_{2 t}\right. \\
& \left.+\left(Y_{2}-\left(1+X_{2}\right) X_{1 t}^{2}+\kappa\left(1+X_{2}\right)^{-2}\right) K\left(1+X_{2}\right)\right\} Y_{1 \theta}
\end{aligned}
$$




$$
\begin{aligned}
+ & \left(1+Z_{1}\right)\left\{4\left(1+X_{2}\right) X_{2 t} Y_{1 t}+6\left(1+X_{2}\right) Y_{1} Y_{2}\right. \\
& \left.+6 X_{2 t}^{2} Y_{1}+6 X_{1 t} X_{2 t} Y_{1}+2\left(1+X_{2}\right) X_{1 t} Y_{2 t}\right\} \\
+ & 2 Z_{1 t}\left(\left(1+X_{2}\right)^{2} Y_{1 t}+4\left(1+X_{2}\right) X_{2 t} Y_{1}+2 X_{1 t} X_{2 t}^{2}+2\left(1+X_{2}\right) X_{1 t} Y_{2}\right) \\
+ & Z_{2}\left\{F_{30}\left(X, X_{t}, Y, Y_{t}\right)-2\left(1+X_{2}\right)\left(X_{1 t} Y_{1 t}+Y_{1}^{2}\right)-4 X_{2 t} X_{1 t} Y_{1}\right. \\
& \left.-Y_{2} X_{1 t}^{2}+6 \kappa X_{2 t}^{2}\left(1+X_{2}\right)^{-4}-2 \kappa Y_{2}\left(1+X_{2}\right)^{-3}\right\} \\
+ & 2 Z_{2 t}\left(Y_{2 t}-X_{2 t} X_{1 t}^{2}-2\left(1+X_{2}\right) X_{1 t} Y_{1}-2 \kappa X_{2 t}\left(1+X_{2}\right)^{-3}\right) \\
+ & \left(Y_{2}-\left(1+X_{2}\right) X_{1 t}^{2}+\kappa\left(1+X_{2}\right)^{-2}\right) F_{11}\left(X, X_{t}, Z, Z_{t}, Y_{1}\right)=0 .
\end{aligned}
$$

It is easy to see that

$$
\begin{aligned}
& \left(\left(1+Z_{1}\right)\left(1+X_{2}\right)^{2}+Z_{2} K\left(1+X_{2}\right)\right)^{-1} \\
= & \left(\left(1+Z_{1}\right)^{2}\left(1+X_{2}\right)^{2}+Z_{2}^{2}\right)^{-1}\left(1+X_{2}\right)^{-2}\left(\left(1+Z_{1}\right)\left(1+X_{2}\right)^{2}-Z_{2} K\left(1+X_{2}\right)\right)+P_{1},
\end{aligned}
$$

where

$$
\begin{aligned}
P_{1}= & \left(\left(1+Z_{1}\right)^{2}\left(1+X_{2}\right)^{2}+Z_{2}^{2}\right)^{-1}\left(1+X_{2}\right)^{-2} Z_{2} \\
& \times\left\{\left[K,\left(1+Z_{1}\right)\left(1+X_{2}\right)^{2}\right]+\left[K,\left(1+X_{2}\right) Z_{2}\right] K+Z_{2}\left(1+X_{2}\right)\left(1+K^{2}\right)\right\}\left(1+X_{2}\right) \\
& \times\left(\left(1+Z_{1}\right)\left(1+X_{2}\right)^{2}+Z_{2} K\left(1+X_{2}\right)\right)^{-1},
\end{aligned}
$$

and that

$$
\begin{aligned}
\left(\left(1+Z_{1}\right)\left(1+X_{2}\right)^{2}-Z_{2} K\left(1+X_{2}\right)\right) \\
\times \\
\quad\left\{\left(1+X_{2}\right)^{2} Y_{1}+2\left(1+X_{2}\right) X_{1 t} X_{2 t}\right. \\
\left.\quad+\left(Y_{2}-\left(1+X_{2}\right) X_{1 t}^{2}+\kappa\left(1+X_{2}\right)^{-2}\right) K\left(1+X_{2}\right)\right\} \\
=\left(1+X_{2}\right)^{2}\left\{\left(1+Z_{1}\right)\left(\left(1+X_{2}\right)^{2} Y_{1}+2\left(1+X_{2}\right) X_{1 t} X_{2 t}\right)\right. \\
\left.\quad+Z_{2}\left(Y_{2}-\left(1+X_{2}\right) X_{1 t}^{2}+\kappa\left(1+X_{2}\right)^{-2}\right)\right\} \\
-\left(1+X_{2}\right)^{2}\left\{\left(1+Z_{1}\right)\left(1+X_{2}\right)\left(Y_{2}-\left(1+X_{2}\right) X_{1 t}^{2}+\kappa\left(1+X_{2}\right)^{-2}\right)\right. \\
\left.\quad-Z_{2}\left(\left(1+X_{2}\right) Y_{1}+2 X_{1 t} X_{2 t}\right)\right\} i \operatorname{sgn} D+P_{2},
\end{aligned}
$$

where

$$
\begin{aligned}
P_{2}= & \left(1+Z_{1}\right)\left(1+X_{2}\right)^{2}\left(Y_{2}-\left(1+X_{2}\right) X_{1 t}^{2}+\kappa\left(1+X_{2}\right)^{-2}\right)\left[K, X_{2}\right] \\
& -Z_{2}\left[K,\left(1+X_{2}\right)^{2}\left(\left(1+X_{2}\right) Y_{1}+2 X_{1 t} X_{2 t}\right)\right] \\
& +\left(1+X_{2}\right)^{2}\left\{\left(1+Z_{1}\right)\left(1+X_{2}\right)\left(Y_{2}-\left(1+X_{2}\right) X_{1 t}^{2}+\kappa\left(1+X_{2}\right)^{-2}\right)\right.
\end{aligned}
$$




$$
\begin{aligned}
& \left.-Z_{2}\left(\left(1+X_{2}\right) Y_{1}+2 X_{1 t} X_{2 t}\right)\right\}(K+i \operatorname{sgn} D) \\
- & Z_{2}\left[K,\left(1+X_{2}\right)\left(Y_{2}-\left(1+X_{2}\right) X_{1 t}^{2}+\kappa\left(1+X_{2}\right)^{-2}\right)\right] K\left(1+X_{2}\right) \\
- & Z_{2}\left(1+X_{2}\right)\left(Y_{2}-\left(1+X_{2}\right) X_{1 t}^{2}+\kappa\left(1+X_{2}\right)^{-2}\right)\left(K^{2}+1\right)\left(1+X_{2}\right) .
\end{aligned}
$$

Therefore, we obtain

$$
\begin{aligned}
& Y_{1 t t}+\left(\left(1+Z_{1}\right)^{2}\left(1+X_{2}\right)^{2}+Z_{2}^{2}\right)^{-1} \\
& \left(\left(1+Z_{1}\right)\left(1+X_{2}\right)^{2} Y_{1}+2\left(1+X_{2}\right) X_{1 t} X_{2 t}\right) \\
& \left.+Z_{2}\left(Y_{2}-\left(1+X_{2}\right) X_{1 t}^{2}+\kappa\left(1+X_{2}\right)^{-2}\right)\right) Y_{1 \theta} \\
& +\left(\left(1+Z_{1}\right)\left(1+X_{2}\right)\left(Y_{2}-\left(1+X_{2}\right) X_{1 t}^{2}+\kappa\left(1+X_{2}\right)^{-2}\right)\right. \\
& \left.\left.-Z_{2}\left(\left(1+X_{2}\right) Y_{1}+2 X_{1 t} X_{2 t}\right)\right)|D| Y_{1}\right\} \\
& =-\left\{\left(\left(1+Z_{1}\right)^{2}\left(1+X_{2}\right)^{2}+Z_{2}^{2}\right)^{-1}\left(1+X_{2}\right)^{-2} P_{2}\right. \\
& +P_{1}\left(\left(1+X_{2}\right)^{2} Y_{1}+2\left(1+X_{2}\right) X_{1 t} X_{2 t}\right. \\
& \left.\left.+\left(Y_{2}-\left(1+X_{2}\right) X_{1 t}^{2}+\kappa\left(1+X_{2}\right)^{-2}\right) K\left(1+X_{2}\right)\right)\right\}(i D) Y_{1} \\
& -\left(\left(1+Z_{1}\right)\left(1+X_{2}\right)^{2}+Z_{2} K\left(1+X_{2}\right)\right)^{-1} \\
& \times\left\{( 1 + Z _ { 1 } ) \left(4\left(1+X_{2}\right) X_{2 t} Y_{1 t}+6\left(1+X_{2}\right) Y_{1} Y_{2}+6 X_{2 t}^{2} Y_{1}\right.\right. \\
& \left.+6 X_{1 t} X_{2 t} Y_{1}+2\left(1+X_{2}\right) X_{1 t} Y_{2 t}\right) \\
& +2 Z_{1 t}\left(\left(1+X_{2}\right)^{2} Y_{1 t}+4\left(1+X_{2}\right) X_{2 t} Y_{1}+2 X_{1 t}^{2} X_{2 t}^{2}+2\left(1+X_{2}\right) X_{1 t} Y_{2}\right) \\
& +Z_{2}\left(F_{30}\left(X, X_{t}, Y, Y_{t}\right)-2\left(1+X_{2}\right)\left(X_{1 t} Y_{1 t}+Y_{1}^{2}\right)-4 X_{2 t} X_{1 t} Y_{1}\right. \\
& \left.-Y_{2} X_{1 t}^{2}+6 \kappa X_{1 t}^{2}\left(1+X_{2}\right)^{-4}-2 \kappa Y_{1}\left(1+X_{2}\right)^{-3}\right) \\
& +2 Z_{2 t}\left(Y_{2 t}-X_{2 t} X_{1 t}^{2}-2\left(1+X_{2}\right) X_{1 t} Y_{1}-2 \kappa X_{2 t}\left(1+X_{2}\right)^{-3}\right) \\
& \left.+\left(Y_{2}-\left(1+X_{2}\right) X_{1 t}^{2}+\kappa\left(1+X_{2}\right)^{-2}\right) F_{11}\left(X, X_{t}, Z, Z_{t}, Y_{1}\right)\right\} \\
& =: f_{1}\left(W, W_{t}^{\prime}\right) \text {, }
\end{aligned}
$$

where $Y_{2 t}, Z_{1 t}$ and $Z_{2 t}$ were replaced by $f_{2}, f_{3}$ and $f_{4}$, respectively. It is a remarkable fact that the coefficient of $Y_{1 \theta}$ in the above equation is identically zero because of (2.9). This is the cancellation stated in the introduction and it makes our problem to be well-posed. Consequently, the required quasi-linear system for $W$ has the form

$$
\left\{\begin{array}{l}
X_{t t}=Y, \quad Y_{1 t t}+a\left(W, X_{t}\right)|D| Y_{1}=f_{1}\left(W, W_{t}^{\prime}\right), \\
Y_{2 t}=f_{2}\left(W, W_{t}^{\prime}\right), \quad Z_{1 t}=f_{3}\left(W, W_{t}^{\prime}\right), \quad Z_{2 t}=f_{4}\left(W, W_{t}^{\prime}\right),
\end{array}\right.
$$


where

$$
\begin{aligned}
a\left(W, X_{t}\right)= & \left(\left(1+Z_{1}\right)^{2}\left(1+X_{2}\right)^{2}+Z_{2}^{2}\right)^{-1} \\
& \times\left\{\left(1+Z_{1}\right)\left(1+X_{2}\right)\left(Y_{2}-\left(1+X_{2}\right) X_{1 t}^{2}+\kappa\left(1+X_{2}\right)^{-2}\right)\right. \\
& \left.\quad-Z_{2}\left(\left(1+X_{2}\right) Y_{1}+2 X_{1 t} X_{2 t}\right)\right\} .
\end{aligned}
$$

Remark 6.1. It holds that

$$
\rho r_{1}\left(\left(1+Z_{1}\right)^{2}\left(1+X_{2}\right)^{2}+Z_{2}^{2}\right)^{1 / 2} a\left(W, X_{t}\right)=-\left.n_{f} \cdot \nabla p\right|_{\Gamma_{t}},
$$

where $n_{f}$ is the unit outward normal vector to the free surface $\Gamma_{t}$.

Remark 6.2. For the non-linear equations (2.15) and (2.16), we can also derive a similar quasi-linear system of equations in the same way as above.

We proceed to investigate the properties of the functions $a$ and $f=\left(f_{1}, \ldots, f_{4}\right)$. Define the operators $P_{3}$ and $P_{4}$ by

$$
\begin{aligned}
P_{3}= & \left\{\left(1+X_{2}\right)^{2} Y_{1}+2\left(1+X_{2}\right) X_{1 t} X_{2 t}\right. \\
& \left.-\left(Y_{2}-\left(1+X_{2}\right) X_{1 t}^{2}+\kappa\left(1+X_{2}\right)^{-2}\right) i \operatorname{sgn} D\left(1+X_{2}\right)\right\}^{-1}, \\
P_{4}= & \left\{\left(1+Z_{1}\right)\left(1+X_{2}\right)^{2}+Z_{2} K\left(1+X_{2}\right)\right\}^{-1} .
\end{aligned}
$$

Lemma 6.1. Let $s \geq 2$. Then for operators $P_{j}, j=1, \ldots, 4$, we have

$$
\left\{\begin{array}{l}
P_{1}\left(X, Z, b, b^{\prime} ; X, Z, b, b^{\prime}\right), P_{2}\left(W, X_{t}, b, b^{\prime} ; X, b, b^{\prime}\right) \in L(2, s ; 2), \\
P_{3}\left(X, X_{t}, Y ; X, X_{t}, Y\right), P_{4}\left(X, Z, b, b^{\prime} ; X, Z, b, b^{\prime}\right) \in L(s, s ; 2)
\end{array}\right.
$$

Proof. From Lemma 5.11, it follows that

$$
\begin{gathered}
Q_{4}\left(X, Z, b, b^{\prime} ; X, b, b^{\prime}\right):=\left(1+Z_{1}\right)\left(1+X_{2}\right)^{2}-1+Z_{2} K\left(X, b, b^{\prime} ; X, b, b^{\prime}\right)\left(1+X_{2}\right) \\
\in L_{0}(q, q ; 2) \quad \text { for } \quad q \geq 2 .
\end{gathered}
$$

Using this fact, the relations

$$
P_{4}=\left(1+Q_{4}\right)^{-1}, \quad T_{\theta} Q_{4}(X, Z, b)=Q_{4}\left(T_{\theta} X, T_{\theta} Z, T_{\theta} b\right) T_{\theta},
$$

and Lemma 3.4, we see that $P_{4}\left(X, Z, b, b^{\prime} ; X, Z, b, b^{\prime}\right) \in L(s, s ; 2)$. Since 


$$
\begin{aligned}
P_{3}= & \kappa^{-1} i \operatorname{sgn} D\left\{1+\left(\left(1+X_{2}\right)^{2} Y_{1}+2\left(1+X_{2}\right) X_{1 t} X_{2 t}\right.\right. \\
& -\left(Y_{2}-\left(1+X_{2}\right) X_{1 t}^{2}+\kappa\left(1+X_{2}\right)^{-2}\right) i \operatorname{sgn} D X_{2} \\
& \left.\left.-\left(Y_{2}-\left(1+X_{2}\right) X_{1 t}^{2}+\kappa\left(\left(1+X_{2}\right)^{-2}-1\right)\right) i \operatorname{sgn} D\right) \kappa^{-1} i \operatorname{sgn} D\right\}^{-1},
\end{aligned}
$$

by the same consideration as above, we obtain $P_{3}\left(X, X_{t}, Y ; X, X_{t}, Y\right) \in L(s, s ; 2)$. By Lemmas 3.2, 3.3 and 5.11, it is not difficult to see that

$$
\left\{\begin{array}{l}
Q_{1}\left(V, X, b, b^{\prime} ; X, b, b^{\prime}\right):=\left[K\left(X, b, b^{\prime} ; X, b, b^{\prime}\right), V\right] \in L_{0}(2, s ; 2), \\
Q_{2}\left(X, b, b^{\prime} ; X, b, b^{\prime}\right):=1+\left(K\left(X, b, b^{\prime} ; X, b, b^{\prime}\right)\right)^{2} \in L(2, s ; 2) .
\end{array}\right.
$$

These facts, together with Lemmas 3.2, 5.11 and the above result for $P_{4}$, show that $P_{1}\left(X, Z, b, b^{\prime} ; X, Z, b, b^{\prime}\right), \quad P_{2}\left(W, X_{t}, b, b^{\prime} ; X, b, b^{\prime}\right) \in L(2, s ; 2)$. The proof is complete.

Making use of Lemmas 3.2, 5.12 and 6.1, we can easily obtain

Lemma 6.2. For any $\beta>1$ and $\kappa>0$, there exists a small positive constant $\varepsilon_{0}=\varepsilon_{0}(\beta, \kappa)$ depending only on $\beta$ and $\kappa$ such that if

$$
\left\{\begin{array}{l}
W, W_{t}^{\prime} \in H^{s}, \quad b \in H^{s+1}, \quad s \geq 3 \\
\|X\|_{3},\|b\|_{3},\|Z\|_{2},\left\|X_{t}\right\|_{2},\|Y\|_{2} \leq \varepsilon_{0}, \quad\|W\|_{s}+\left\|W_{t}^{\prime}\right\|_{s}+\|b\|_{s+1} \leq c, \quad c>0
\end{array}\right.
$$

then $a\left(W, X_{t}\right)-\kappa, f\left(W, W_{t}^{\prime}\right) \in H^{s}$ and

$$
2^{-1} \kappa \leq a\left(W, X_{t}\right) \leq 2 \kappa, \quad\left\|f\left(W, W_{t}^{\prime}\right)\right\|_{s} \leq C\left(\|W\|_{s}+\left\|W_{t}^{\prime}\right\|_{s}\right) .
$$

Moreover, for $W^{0}$ and $W_{t}^{0^{\prime}}$ satisfying (6.8) it holds that

$$
\begin{gathered}
\left\|a\left(W, X_{t}\right)-a\left(W^{0}, X_{t}^{0}\right)\right\|_{s} \leq C\left(\left\|W-W^{0}\right\|_{s}+\left\|X_{t}-X_{t}^{0}\right\|_{s}\right), \\
\left\|f\left(W, W_{t}^{\prime}\right)-f\left(W^{0}, W_{t}^{0^{\prime}}\right)\right\|_{s} \leq C\left(\left\|W-W^{0}\right\|_{s}+\left\|W_{t}^{\prime}-W_{t}^{0^{\prime}}\right\|_{s}\right),
\end{gathered}
$$

where $C=C(\beta, \kappa, s, c)>0$.

Next, assuming that $X$ satisfies (2.9)-(2.11) we determine the initial values $\bar{W}=\left.W\right|_{t=0}$ and $\bar{W}_{t}^{\prime}=\left.W_{t}^{\prime}\right|_{t=0}$ from the initial data $U$ and $V$. It is clear that $\bar{X}=U, \bar{Z}=U_{\theta}, \bar{X}_{1 t}=V$ and $\bar{X}_{2 t}=K(U)\left(1+U_{2}\right) V . \quad$ By virtue of (6.2) with $k=1$, we have

$$
Y_{2}=K(X)\left(1+X_{2}\right) Y_{1}+F_{10}\left(X, X_{t}\right)
$$


Substituting this into (2.9), we see that

$$
\begin{aligned}
\bar{Y}_{1}=- & \left\{\left(1+\bar{Z}_{1}\right)\left(1+\bar{X}_{2}\right)^{2}+\bar{Z}_{2} K(\bar{X})\left(1+\bar{X}_{2}\right)\right\}^{-1} \\
\times & \left\{2\left(1+\bar{Z}_{1}\right)\left(1+\bar{X}_{2}\right) \bar{X}_{1 t} \bar{X}_{2 t}\right. \\
& \left.+\bar{Z}_{2}\left(F_{10}\left(\bar{X}, \bar{X}_{t}\right)-\left(1+\bar{X}_{2}\right) \bar{X}_{1 t}^{2}+\kappa\left(1+\bar{X}_{2}\right)^{-2}\right)\right\} .
\end{aligned}
$$

This and (6.9) imply that $\bar{Y}_{2}=K(\bar{X})\left(1+\bar{X}_{2}\right) \bar{Y}_{1}+F_{10}\left(\bar{X}, \bar{X}_{t}\right)$. Eliminating $Y_{2 t}$ from (6.4) and (6.5), we obtain

$$
\begin{aligned}
\bar{Y}_{1 t}= & -\left\{\left(1+\bar{Z}_{1}\right)\left(1+\bar{X}_{2}\right)^{2}+\bar{Z}_{2} K(\bar{X})\left(1+\bar{X}_{2}\right)\right\}^{-1} \\
& \times\left\{\left(\partial_{\theta} \bar{X}_{1 t}\right)\left(\left(1+\bar{X}_{2}\right)^{2} \bar{Y}_{1}+2\left(1+\bar{X}_{2}\right) \bar{X}_{1 t} \bar{X}_{2 t}\right)\right. \\
& +\left(\partial_{\theta} \bar{X}_{2 t}\right)\left(\bar{Y}_{2}-\left(1+\bar{X}_{2}\right) \bar{X}_{1 t}^{2}+\kappa\left(1+\bar{X}_{2}\right)^{-2}\right) \\
& +\left(1+\bar{Z}_{1}\right)\left(4\left(1+\bar{X}_{2}\right) \bar{X}_{2 t} \bar{Y}_{1}+2 \bar{X}_{1 t} \bar{X}_{2 t}^{2}+2\left(1+\bar{X}_{2}\right) \bar{X}_{1 t} \bar{Y}_{2}\right) \\
& \left.+\bar{Z}_{2}\left(F_{20}\left(\bar{X}, \bar{X}_{t}, \bar{Y}\right)-\bar{X}_{2 t} \bar{X}_{1 t}^{2}-2\left(1+\bar{X}_{2}\right) \bar{X}_{1 t} \bar{Y}_{1}-2 \kappa \bar{X}_{2 t}\left(1+\bar{X}_{2}\right)^{-3}\right)\right\}
\end{aligned}
$$

For $\bar{W}$ and $\bar{W}_{t}^{\prime}$ defined above, we get the following lemma as a consequence of Lemmas 5.12 and 6.1 .

Lemma 6.3. For any $\beta>1$, there exists a small positive constant $\varepsilon_{1}=\varepsilon_{1}(\beta)$ depending only on $\beta$ such that if

$$
\left\{\begin{array}{l}
U \in H^{s+1 / 2}, \quad V, b \in H^{s}, \quad s \geq 3+1 / 2, \\
\|U\|_{3},\|b\|_{3} \leq \varepsilon_{1},\|U\|_{s}+\|V\|_{s}+\|b\|_{s} \leq c, \quad c>0
\end{array}\right.
$$

then

$$
\|\bar{X}\|_{s+1 / 2}+\left\|\bar{X}_{t}\right\|_{s}+\|\bar{Y}\|_{s-1 / 2}+\left\|\bar{Y}_{1 t}\right\|_{s-1}+\|\bar{Z}\|_{s-1 / 2} \leq C\left(\|U\|_{s+1 / 2}+\|V\|_{s}\right),
$$

where $C=C(\beta, \kappa, s, c)>0$. Moreover, if we assume, in place of (6.10), that

$$
U, b \in H^{3}, \quad V \in H^{2}, \quad\|U\|_{3},\|b\|_{3} \leq \varepsilon_{1}, \quad\|V\|_{2} \leq c, \quad c>0,
$$

then

$$
\|\bar{X}\|_{3}+\|\bar{Z}\|_{2}+\left\|\bar{X}_{t}\right\|_{2}+\|\bar{Y}\|_{2} \leq C\left(\|U\|_{3}+\|V\|_{2}\right),
$$

where $C=C(\beta, \kappa, c)>0$. 


\section{$\S 7 . \quad$ Proof of Theorems 1 and 2}

The proof of Theorem 2 is carried out in the same way as that of Theorem 1. Therefore, in this section we only give the proof of Theorem 1 .

Assuming that the condition (2.12) is fulfilled, we define $\bar{W}$ and $\bar{W}_{t}^{\prime}$ from $U, V$ and $b$ according to the previous section. If we chose $\delta$ suitably small, then the quasi-linear system (6.7) under the initial conditions $\left.W\right|_{t=0}=\bar{W}$ and $\left.\bar{W}_{t}^{\prime}\right|_{t=0}=\bar{W}_{t}^{\prime}$ has a unique solution $W=(X, Y, Z)$ satisfying

$$
\left\{\begin{array}{l}
X \in C^{2}\left([0, T] ; H^{s-1}\right), \quad Y_{2}, Z \in C^{1}\left([0, T] ; H^{s-1}\right), \\
Y_{1} \in C^{j}\left([0, T] ; H^{s-1 / 2-j / 2}\right), \quad j=0,1,2,
\end{array}\right.
$$

for some time interval $[0, T]$, because of Lemmas 6.2 and 6.3. The proof of this fact is standard (for example, see [7, section 6]), so that we omit it. We proceed to show that $X$ is the desired solution. By the definition of $\bar{Y}$, equation

$$
\begin{aligned}
& \left(1+X_{2}\right)\left(1+Z_{1}\right)\left(\left(1+X_{2}\right) Y_{1}+2 X_{1 t} X_{2 t}\right) \\
& \quad+Z_{2}\left(Y_{2}-\left(1+X_{2}\right) X_{1 t}^{2}+\kappa\left(1+X_{2}\right)^{-2}\right)=0
\end{aligned}
$$

holds for $t=0$. The equations for $Z$ and $X$ in (6.7) imply that the time derivative for the left hand side of (7.2) is identically zero for $0 \leq t \leq T$. Therefore, (7.2) is also valid for $0 \leq t \leq T$, and it is sufficient to show that $Z=X_{\theta}$ and (2.10), because we have $Y=X_{t t}$. The equation for $Y_{2}$ in (6.7) can be rewritten in the form $\left(X_{2 t}-K\left(1+X_{2}\right) X_{1 t}\right)_{t t}=0$, and it is clear that $\left.\partial_{t}^{j}\left(X_{2 t}-K\left(1+X_{2}\right) X_{1 t}\right)\right|_{t=0}=0$ for $j=0,1$. Hence, (2.10) is shown. Differentiating (2.10), we have

$$
\left\{\begin{array}{l}
Y_{2 t}=K\left(1+X_{2}\right) Y_{1 t}+F_{20}\left(X, X_{t}, Y\right), \\
Y_{2 t t}=K\left(1+X_{2}\right) Y_{1 t t}+F_{30}\left(X, X_{t}, Y, Y_{t}\right), \\
X_{2 t \theta}=K\left(1+X_{2}\right) X_{1 t \theta}+F_{01}\left(X, X_{\theta}, X_{1 t}\right) .
\end{array}\right.
$$

Differentiating (7.2) with respect to $t$, we see that (6.5) is valid. The equation for $Z_{1}$ in (6.7) can be rewritten as the equation (6.5) with $Z_{2 t}$ replaced by

$$
-i \operatorname{sgn} D\left(1+X_{2}\right) Z_{1 t}+(i \operatorname{sgn} D+K)\left(1+X_{2}\right) X_{1 t \theta}+F_{01}\left(X, Z, X_{1 t}\right) \text {. }
$$

Subtracting such an equation from the equation (6.5) itself, we obtain

$$
Z_{2 t}=-i \operatorname{sgn} D\left(1+X_{2}\right)\left(Z_{1}-X_{1 \theta}\right)_{t}+K\left(1+X_{2}\right) X_{1 t \theta}+F_{01}\left(X, Z, X_{1 t}\right)
$$


This and the last equation in (7.3) yield that

$$
\left(Z_{2}-X_{2 \theta}\right)_{t}=-i \operatorname{sgn} D\left(1+X_{2}\right)\left(Z_{1}-X_{1 \theta}\right)_{t}+F_{01}\left(X, Z, X_{1 t}\right)-F_{01}\left(X, X_{\theta}, X_{1 t}\right) \text {. }
$$

If we subtract the equation obtained by differentiating (7.2) two times with respect to $t$ from a similar equation derived from the equation for $Y_{1}$ in (6.7) by taking (7.2) into account and use the second equation in (7.3), then

$$
\begin{aligned}
& \left(Z_{1}-X_{1 \theta}\right)_{t t}\left(\left(1+X_{2}\right)^{2} Y_{1}+2\left(1+X_{2}\right) X_{1 t} X_{2 t}\right) \\
& +\left(Z_{2 t t}-\left(K\left(1+X_{2}\right) Y_{1 \theta}+F_{11}\left(X, X_{t}, Z, Z_{t}, Y_{1}\right)\right)\right) \\
& \quad \times\left(Y_{2}-\left(1+X_{2}\right) X_{1 t}^{2}+\kappa\left(1+X_{2}\right)^{-2}\right)=0 .
\end{aligned}
$$

Differentiating (7.4) with respect to $t$ and putting the result into the above equation to eliminate $Z_{2 t t}$, we see that

$$
\begin{aligned}
&\left(Z_{1}-X_{1 \theta}\right)_{t t}=\left\{\left(1+X_{2}\right)^{2} Y_{1}+2\left(1+X_{2}\right) X_{1 t} X_{2 t}\right. \\
&\left.-\left(Y_{2}-\left(1+X_{2}\right) X_{1 t}^{2}+\kappa\left(1+X_{2}\right)^{-2}\right) i \operatorname{sgn} D\left(1+X_{2}\right)\right\}^{-1} \\
& \times\left(Y_{2}-\left(1+X_{2}\right) X_{1 t}^{2}+\kappa\left(1+X_{2}\right)^{-2}\right) K_{1,1,0}\left(X, X_{t}\right)\left(Z_{1}-X_{1 \theta}\right)_{t}
\end{aligned}
$$

On the other hand, by the definition of $Y_{1 t}$, the equation (6.5) holds at $t=0$, if $Z_{1 t}, Z_{2 t}$ and $Y_{2 t}$ are replaced by $X_{1 t \theta}, X_{2 t \theta}$ and $K\left(1+X_{2}\right) Y_{1 t}+F_{20}\left(X, X_{t}, Y\right)$, respectively. Subtracting such an equation from the equation (6.5) itself and using the first equation in (7.3), we get

$$
\begin{aligned}
& \left(Z_{1}-X_{1 \theta}\right)_{t}\left(\left(1+X_{2}\right)^{2} Y_{1}+2\left(1+X_{2}\right) X_{1 t} X_{2 t}\right) \\
& \quad+\left(Z_{2}-X_{2 \theta}\right)_{t}\left(Y_{2}-\left(1+X_{2}\right) X_{1 t}^{2}+\kappa\left(1+X_{2}\right)^{-2}\right)=0 \text { at } t=0 .
\end{aligned}
$$

Putting (7.5) into this relation, we obtain $\left.\left(Z_{1}-X_{1 \theta}\right)_{t}\right|_{t=0}=0$. It is clear that $Z-\left.X_{\theta}\right|_{t=0}=0$. These facts together with (7.5) and (7.6) imply that $Z=X_{\theta}$. Hence, it has shown that $X$ is a solution of (2.9)-(2.11). Note that

$$
\left\{\begin{array}{l}
X_{1 t t}=Y \in C^{j}\left([0, T] ; H^{s-1 / 2-j / 2}\right), \quad j=0,1,2, \\
X_{2 t t}=K\left(1+X_{2}\right) Y_{1}+F_{10}\left(X, X_{t}, Y\right) \in C^{j}\left([0, T] ; H^{s-1 / 2-j / 2}\right), \quad j=0,1,2, \\
X_{\theta}=Z \in C^{1}\left([0, T] ; H^{s-1}\right) .
\end{array}\right.
$$

These relations yield that $X$ satisfies (2.13). The uniqueness of the solution comes from that of the corresponding initial value problem for the quasi-linear system (6.7). Finally, the continuous dependence of solutions on the initial 
data can be proved by almost the same way as in [1, section 5], so that we omit it. The proof is complete.

\section{§. Proof of Theorem 3}

Assuming that $X$ satisfies (2.15), (2.16) and (2.11) with $U=0$, we derive an equation of an evolution type for $X$. Differentiating (2.16) with respect to $t$, we obtain

$$
X_{2 t t}=K\left(1+X_{2}\right) X_{1 t t}+K X_{2 t}\left(\alpha+X_{1 t}\right)+K_{1,1,0}\left(1+X_{2}\right)\left(\alpha+X_{1 t}\right) .
$$

Putting this into (2.15), we see that

$$
\begin{aligned}
& X_{1 t t}+2 \alpha X_{2 t}-\left(\alpha^{2}-\kappa\right) X_{2 \theta} \\
& \begin{aligned}
= & \left.\left(1+X_{1 \theta}\right)\left(1+X_{2}\right)^{2}+X_{2 \theta} K\left(1+X_{2}\right)\right\}^{-1} \\
& \times\left\{\left(\left(1+X_{1 \theta}\right)\left(1+X_{2}\right)^{2}-1+X_{2 \theta} K\left(1+X_{2}\right)\right)\left(2 \alpha X_{2 t}-\left(\alpha^{2}-\kappa\right) X_{2 \theta}\right)\right. \\
& \quad-2 \alpha\left(\left(1+X_{2}\right)\left(1+X_{1 \theta}\right)-1\right) X_{2 t}-2\left(1+X_{2}\right)\left(1+X_{1 \theta}\right) X_{1 t} X_{2 t} \\
& \quad-X_{2 \theta}\left(K X_{2 t}\left(\alpha+X_{1 t}\right)+K_{1,1,0}\left(1+X_{2}\right)\left(\alpha+X_{1 t}\right)\right. \\
\quad & \left.\left.\quad\left(\left(1+X_{2}\right)\left(\alpha+X_{1 t}\right)^{2}-\alpha^{2}\right)+\kappa\left(\left(1+X_{2}\right)^{-2}-1\right)\right)\right\} \\
= & g_{3}\left(X, X_{t}\right) .
\end{aligned}
\end{aligned}
$$

On the other hand, according to Remark 4.1 we can rewrite (2.16) as

$$
X_{2 t}=K\left(\left(1+X_{2}\right)\left(\alpha+X_{1 t}\right)-\alpha\right)+\alpha K\left(1+X_{2}\right)^{-1} X_{2} .
$$

The equation (8.2) differentiated with respect to $t$ and (8.1) yield that

$$
\begin{aligned}
& X_{2 t t}-\left(\alpha^{2}-\kappa\right) K_{0} X_{2 \theta} \\
&= K\left(1+X_{2}\right) g_{3}\left(X, X_{t}\right)-K X_{2}\left(2 \alpha X_{2 t}-\left(\alpha^{2}-\kappa\right) X_{2 \theta}\right) \\
&-K\left(X_{2 t} X_{1 t}+\alpha\left(\left(1+X_{2}\right)^{-2}-1\right) X_{2 t}\right)+\left(\alpha^{2}-\kappa\right) K_{1} X_{2 \theta} \\
&-K_{1,1,0}\left\{\left(\left(1+X_{2}\right)\left(\alpha+X_{1 t}\right)-\alpha\right)+\alpha\left(1+X_{2}\right)^{-1} X_{2}\right\} \\
&=: g_{2}\left(X, X_{t}\right) .
\end{aligned}
$$

Differentiating (8.2) with respect to $\theta$ and using the relation $X_{2 \theta}=\left(\alpha^{2}-\kappa\right)^{-1}\left(X_{1 t t}\right.$ $\left.+2 \alpha X_{2 t}-g_{3}\left(X, X_{t}\right)\right)$, we get

$$
X_{2 \theta t}=\left(K\left(1+X_{2}\right) X_{1 \theta}+2 \alpha\left(\alpha^{2}-\kappa\right)^{-1} K_{0}\left(X_{1 t}+2 \alpha X_{2}\right)\right)_{t}+\left(\alpha^{2}-\kappa\right)^{-1} h_{1}\left(X, X_{t}\right),
$$


where

$$
\begin{aligned}
h_{1}\left(X, X_{t}\right)= & -2 \alpha K_{0} g_{3}\left(X, X_{t}\right)+\left(\alpha^{2}-\kappa\right)\left\{-K X_{2 t} X_{1 t}-K_{1,1,0}\left(1+X_{2}\right) X_{1 \theta}\right. \\
& +2 \alpha K_{1} X_{2 \theta}+K X_{2 \theta} X_{1 t}+K_{1.0,1}\left(\left(1+X_{2}\right)\left(\alpha+X_{1 t}\right)-\alpha\right) \\
& \left.+\alpha K\left(\left(1+X_{2}\right)^{-2}-1\right) X_{2 \theta}+\alpha K_{1,0,1}\left(1+X_{2}\right)^{-1} X_{2}\right\} .
\end{aligned}
$$

Hence,

$$
\begin{aligned}
X_{2 \theta}= & K\left(1+X_{2}\right) X_{1 \theta}+2 \alpha\left(\alpha^{2}-\kappa\right)^{-1} K_{0}\left(X_{1 t}-V+2 \alpha X_{2}\right) \\
& +\left(\alpha^{2}-\kappa\right)^{-1} \int_{0}^{t} h_{1}\left(X, X_{t}\right) d \tau .
\end{aligned}
$$

Putting (8.2) and (8.3) into (8.1), we obtain

$$
X_{1 t t}-\left(\alpha^{2}-\kappa\right) K_{0} X_{1 \theta}=-2 \alpha K_{0} V+g_{1}\left(X, X_{t}\right)+\int_{0}^{t} h_{1}\left(X, X_{t}\right) d \tau,
$$

where

$$
\begin{array}{r}
g_{1}\left(X, X_{t}\right)=g_{3}\left(X, X_{t}\right)+\left(\alpha^{2}-\kappa\right)\left(K_{0} X_{2} X_{1 \theta}+K_{1}\left(1+X_{2}\right) X_{1 \theta}\right) \\
-2 \alpha K_{0}\left\{K_{0} X_{2} X_{1 t}+K_{1}\left(\left(1+X_{2}\right)\left(\alpha+X_{1 t}\right)-\alpha\right)\right. \\
\left.-\alpha K_{0}\left(1+X_{2}\right)^{-1} X_{2}^{2}+\alpha K_{1}\left(1+X_{2}\right)^{-1} X_{2}\right\} .
\end{array}
$$

Therefore, the desired evolution equation for $X$ has the form

$$
X_{t t}-\left(\alpha^{2}-\kappa\right) K_{0} X_{\theta}=W+g\left(X, X_{t}\right)+\int_{0}^{t} h\left(X, X_{t}\right) d \tau
$$

with initial conditions

$$
\left.X\right|_{t=0}=0,\left.\quad X\right|_{t=0}=\left(V, K_{0} V\right),
$$

where $W=\left(-2 \alpha K_{0} V, 0\right), g=\left(g_{1}, g_{2}\right)$ and $h=\left(h_{1}, 0\right)$.

As a result of Lemmas 5.12 and 6.1 , we can prove

Lemma 8.1. For any $\beta>1$, there exists a small positive constant $\varepsilon_{2}=\varepsilon_{2}(\beta)$ depending only on $\beta$ such that if

$$
X \in H^{3}, \quad X_{t} \in H^{2}, \quad\|X\|_{3} \leq \varepsilon_{2}, \quad\left\|X_{t}\right\|_{2} \leq c, \quad c>0,
$$

then 


$$
\left\|g\left(X, X_{t}\right)\right\|_{2}+\left\|h\left(X, X_{t}\right)\right\|_{2} \leq C\left(\|X\|_{3}+\left\|X_{t}\right\|_{2}\right)^{2}
$$

where $C=C(\beta, \alpha, \kappa, c)>0$.

In view of (8.4) and Lemma 3.2, it is natural to consider the equation

$$
u_{t t}-\omega|D| u=f
$$

where $\omega$ is a positive constant. Fix an arbitrary number $n$ and define projections $P^{(1)}$ and $P^{(2)}$ by

$$
P^{(1)} u(\theta)=\sum_{|m| \geq n} u_{m} \mathrm{e}^{i m \theta} \quad \text { for } \quad u(\theta)=\sum_{m=-\infty}^{\infty} u_{m} \mathrm{e}^{i m \theta}, \quad P^{(2)}=1-P^{(1)} .
$$

Using these projections, we decompose a solution $u$ of (8.6) as

$$
u=u^{(1)}+u^{(2)}, \quad u^{(j)}=P^{(j)} u, j=1,2
$$

Then, it is easy to see that

$$
\left\||D|^{\lambda} u^{(1)}\right\|_{s} \geq n^{\lambda}\left\|u^{(1)}\right\|_{s}, \quad\left\||D|^{\lambda} u^{(2)}\right\|_{s} \leq n^{\lambda}\left\|u^{(2)}\right\|_{s}
$$

for any $\lambda>0$ and $s \in \mathbf{R}^{1}$, and that

$$
u_{t t}^{(j)}-\omega|D| u^{(j)}=P^{(j)} f, \quad j=1,2 .
$$

In the following, we fix a real number $p$ such that $1<p<2$. Since

$$
\begin{aligned}
\frac{1}{2} \frac{d}{d t}\left\|u_{t}^{(1)} \pm \sqrt{\omega|D|} u^{(1)}\right\|_{2}^{2}= & \pm\left\|(\omega|D|)^{1 / 4}\left(u_{t}^{(1)} \pm \sqrt{\omega|D|} u^{(1)}\right)\right\|_{2}^{2} \\
& +\left(f, u_{t}^{(1)} \pm \sqrt{\omega|D|} u^{(1)}\right)_{2},
\end{aligned}
$$

where $(\cdot, \cdot)_{2}$ is the inner product in $H^{2}$, we have

$$
\begin{gathered}
\frac{1}{2} \frac{d}{d t}\left\|u_{t}^{(1)}+\sqrt{\omega|D|} u^{(1)}\right\|_{2}^{2} \geq \sqrt{\omega n}\left\|u_{t}^{(1)}+\sqrt{\omega|D|} u^{(1)}\right\|_{2}^{2}-\|f\|_{2}\left\|u_{t}^{(1)}+\sqrt{\omega|D|} u^{(1)}\right\|_{2} \\
\frac{1}{2} \frac{d}{d t}\left\|u_{t}^{(1)}-\sqrt{\omega|D|} u^{(1)}\right\|_{2}^{2} \leq-\sqrt{\omega n}\left\|u_{t}^{(1)}-\sqrt{\omega|D|} u^{(1)}\right\|_{2}^{2}+\|f\|_{2}\left\|u_{t}^{(1)}-\sqrt{\omega|D|} u^{(1)}\right\|_{2} .
\end{gathered}
$$

These inequalities, the identity

$$
\frac{d}{d t}\left\|u_{t}^{(1)} \pm \sqrt{\omega|D|} u^{(1)}\right\|_{2}^{p}=\frac{p}{2}\left\|u_{t}^{(1)} \pm \sqrt{\omega|D|} u^{(1)}\right\|_{2}^{p-2} \frac{d}{d t}\left\|u_{t}^{(1)} \pm \sqrt{\omega|D|} u^{(1)}\right\|_{2}^{2}
$$


and Young's inequality imply that

$$
\begin{gathered}
\frac{d}{d t}\left\|u_{t}^{(1)}+\sqrt{\omega|D|} u^{(1)}\right\|_{2}^{p} \geq(p \sqrt{\omega n}-1)\left\|u_{t}^{(1)}+\sqrt{\omega|D|} u^{(1)}\right\|_{2}^{p}-p\|f\|_{2}^{p}, \\
\frac{d}{d t}\left\|u_{t}^{(1)}-\sqrt{\omega|D|} u^{(1)}\right\|_{2}^{p} \leq-(p \sqrt{\omega n}-1)\left\|u_{t}^{(1)}-\sqrt{\omega|D|} u^{(1)}\right\|_{2}^{p}+p\|f\|_{2}^{p} .
\end{gathered}
$$

Similarly, we obtain

$$
\begin{aligned}
\frac{d}{d t}\left(\left\|u_{t}^{(2)}\right\|_{2}\right. & \left.+\left\|\sqrt{\omega|D|} u^{(2)}\right\|_{2}+\left\|u^{(2)}\right\|_{2}\right) \\
& \leq(\sqrt{\omega n}+1)\left(\left\|u_{t}^{(2)}\right\|_{2}+\left\|\sqrt{\omega|D|} u^{(2)}\right\|_{2}\right)+\|f\|_{2} .
\end{aligned}
$$

Using these estimates, we show the following

Lemma 8.2. Let $\varepsilon_{2}$ be the constant in Lemma 8.1 and $M, T>0$. There exists a large number $N_{1}=N_{1}(\beta, \alpha, \kappa, M, T)$ such that if $n \geq N_{1}, X \in C^{1}([0, T]$; $H^{4+1 / 2}$ ) satisfies (2.15), (2.16), (2.11) and

$$
\|X(t)\|_{3} \leq \varepsilon_{2},\left\|X_{t}(t)\right\|_{4+1 / 2}+\|X(t)\|_{4+1 / 2} \leq M \quad \text { for } \quad 0 \leq t \leq T,
$$

and the initial data in (2.11) satisfy $U=0$ and $P^{(1)} V=V$, then the estimate

$$
\left\|X_{t}(t)+\sqrt{\left(\alpha^{2}-\kappa\right)|D|} X(t)\right\|_{2} \geq \frac{1}{4}\|V\|_{2} \exp \left(\frac{1}{2} \sqrt{\left(\alpha^{2}-\kappa\right) n} t\right)
$$

holds for $0 \leq t \leq T$.

Proof. We decompose $X$ into $X^{(1)}$ and $X^{(2)}$ according to the decomposition (8.7). Since $X$ satisfies (8.4), by the preceding arguments we see that

$$
\begin{aligned}
& \frac{d}{d t}\left\|X_{t}^{(1)}+\sqrt{\left(\alpha^{2}-\kappa\right)|D|} X^{(1)}\right\|_{2}^{p} \\
& \geq\left(p \sqrt{\left(\alpha^{2}-\kappa\right) n}-1\right)\left\|X_{t}^{(1)}+\sqrt{\left(\alpha^{2}-\kappa\right)|D|} X^{(1)}\right\|_{2}^{p} \\
& -p\left(\left\|\left(\alpha^{2}-\kappa\right)\left(K_{0}+i \operatorname{sgn} D\right)(i D) X^{(1)}\right\|_{2}^{p}\right. \\
& \left.\quad+\|W\|_{2}^{p}+\left\|g\left(X, X_{t}\right)\right\|_{2}^{p}+t^{p-1} \int_{0}^{t}\left\|h\left(X, X_{t}\right)\right\|_{2}^{p} d \tau\right),
\end{aligned}
$$


(8.9) $\quad \frac{d}{d t}\left\|X_{t}^{(1)}-\sqrt{\left(\alpha^{2}-\kappa\right)|D|} X^{(1)}\right\|_{2}^{p}$

$$
\begin{aligned}
\leq- & \left(p \sqrt{\left(\alpha^{2}-\kappa\right) n}-1\right)\left\|X_{t}^{(1)}-\sqrt{\left(\alpha^{2}-\kappa\right)|D|} X^{(1)}\right\|_{2}^{p} \\
+ & p\left(\left\|\left(\alpha^{2}-\kappa\right)\left(K_{0}+i \operatorname{sgn} D\right)(i D) X^{(1)}\right\|_{2}^{p}\right. \\
& \left.+\|W\|_{2}^{p}+\left\|g\left(X, X_{t}\right)\right\|_{2}^{p}+t^{p-1} \int_{0}^{t}\left\|h\left(X, X_{t}\right)\right\|_{2}^{p} d \tau\right),
\end{aligned}
$$

$$
\begin{aligned}
& \frac{d}{d t}\left(\left\|X_{t}^{(2)}\right\|_{2}+\left\|\sqrt{\left(\alpha^{2}-\kappa\right)|D|} X^{(2)}\right\|_{2}+\left\|X^{(2)}\right\|_{2}\right) \\
& \leq\left(\sqrt{\left(\alpha^{2}-\kappa\right) n}+1\right)\left(\left\|X_{t}^{(2)}\right\|_{2}+\left\|\sqrt{\left(\alpha^{2}-\kappa\right)|D|} X^{(2)}\right\|_{2}\right) \\
& \quad+\left\|\left(\alpha^{2}-\kappa\right)\left(K_{0}+i \operatorname{sgn} D\right)(i D) X^{(2)}\right\|_{2}+\left\|g\left(X, X_{t}\right)\right\|_{2}+\int_{0}^{t}\left\|h\left(X, X_{t}\right)\right\|_{2} d \tau .
\end{aligned}
$$

Define $E_{ \pm}(t)$ by

$$
\begin{aligned}
E_{ \pm}(t)= & 2\left\|X_{t}^{(1)}+\sqrt{\left(\alpha^{2}-\kappa\right)|D|} X^{(1)}\right\|_{2}^{p} \pm\left\|X_{t}^{(1)}-\sqrt{\left(\alpha^{2}-\kappa\right)|D|} X^{(1)}\right\|_{2}^{p} \\
& \pm\left(\left\|X_{t}^{(2)}\right\|_{2}+\left\|\sqrt{\left(\alpha^{2}-\kappa\right)|D|} X^{(2)}\right\|_{2}+\left\|X^{(2)}\right\|_{2}\right) .
\end{aligned}
$$

Then, it follows from (8.8)-(8.10) that

$$
\begin{aligned}
\frac{d}{d t} E_{-}(t) \geq & \frac{p+1}{2} \sqrt{\left(\alpha^{2}-\kappa\right) n} E_{-}(t)+\left(\frac{p-1}{2} \sqrt{\left(\alpha^{2}-\kappa\right) n}-1\right) E_{+}(t) \\
- & 3 p\left(\left\|\left(\alpha^{2}-\kappa\right)\left(K_{0}+i \operatorname{sgn} D\right)(i D) X^{(1)}\right\|_{2}^{p}\right. \\
& \left.+\|W\|_{2}^{p}+\left\|g\left(X, X_{t}\right)\right\|_{2}^{p}+t^{p-1} \int_{0}^{t}\left\|h\left(X, X_{t}\right)\right\|_{2}^{p} d \tau\right) \\
& -\left(\left\|\left(\alpha^{2}-\kappa\right)\left(K_{0}+i \operatorname{sgn} D\right)(i D) X^{(2)}\right\|_{2}\right. \\
& \left.+\left\|X^{(2)}\right\|_{2}+\left\|g\left(X, X_{t}\right)\right\|_{2}+\int_{0}^{t}\left\|h\left(X, X_{t}\right)\right\|_{2} d \tau\right) .
\end{aligned}
$$

By Lemma 3.2, we have 


$$
\left\|\left(K_{0}+i \operatorname{sgn} D\right)(i D) X^{(1)}\right\|_{2}^{p}+\left\|\left(K_{0}+i \operatorname{sgn} D\right)(i D) X^{(2)}\right\|_{2} \leq C E_{+}(t)
$$

with a constant $C$ independent of $n$. By Lemma 8.1 and the interpolation inequality $\|u\|_{3} \leq\|u\|_{3+1 /(2-p)}^{1-p / 2}\|u\|_{2+1 / 2}^{p / 2}$, we get

$$
\left\|g\left(X, X_{t}\right)\right\|_{2}+\left\|h\left(X, X_{t}\right)\right\|_{2} \leq C\left(\left\|X_{t}\right\|_{2}+\|X\|_{3+, 1 /(2-p)}\right)^{2-p} E_{+}(t) .
$$

Putting $p=4 / 3$ and combining (8.11)-(8.13), we see that

$$
\begin{aligned}
& \frac{d}{d t} E_{-}(t) \geq \frac{p+1}{2} \sqrt{\left(\alpha^{2}-\kappa\right) n} E_{-}(t)-3 p\|W\|_{2}^{p} \\
&+\left(\frac{p-1}{2} \sqrt{\left(\alpha^{2}-\kappa\right) n}-C\right) E_{+}(t)-C \int_{0}^{t} E_{+}(\tau) d \tau,
\end{aligned}
$$

where $C$ is independent of $n$. Therefore, by Gronwall's inequality there exists a large number $N_{0}$ such that if $n \geq N_{0}$, then

$$
\begin{aligned}
E_{-}(t) \geq & E_{-}(0) \exp \left(\frac{p+1}{2} \sqrt{\left(\alpha^{2}-\kappa\right) n} t\right) \\
& -3 p\|W\|_{2}^{p} \int_{0}^{t} \exp \left(\frac{p+1}{2} \sqrt{\left(\alpha^{2}-\kappa\right) n}(t-\tau)\right) d \tau \\
\geq & \left(1-\frac{C}{\sqrt{n}}\right)\|V\|_{2}^{p} \exp \left(\frac{p+1}{2} \sqrt{\left(\alpha^{2}-\kappa\right) n t}\right) .
\end{aligned}
$$

Hence, we obtain the desired estimate. The proof is complete.

Now, we can easily prove Theorem 3. In fact, if we assume that the statement in Theorem 3 is true and apply the estimate in Lemma 8.2 to $X^{(n)}$, then for $t \in(0, T]$ it holds that

$$
\begin{aligned}
& \left\|X_{t}^{(n)}(t)+\sqrt{\left(\alpha^{2}-\kappa\right)|D|} X^{(n)}(t)\right\|_{2} \geq \frac{1}{4}\left\|\mathrm{e}^{-n^{1 / 4}+i n \theta}\right\|_{2} \exp \left(\frac{1}{2} \sqrt{\left(\alpha^{2}-\kappa\right) n t}\right) \\
& =\frac{\sqrt{2 \pi}}{4}(1+n)^{2} \exp \left(\frac{1}{2} \sqrt{\left(\alpha^{2}-\kappa\right) n} t-n^{1 / 4}\right) \\
& \rightarrow \infty \quad \text { as } \quad n \rightarrow \infty .
\end{aligned}
$$

This contradicts (2.21). Theorem 3 is proved. 


\section{References}

[1] Iguchi, T., Tanaka, N. and Tani, A., On the two-phase free boundary problem for two-dimensional water waves, Math. Ann., 309 (1997), 199-223.

[2] Nalimov, V. I., The Cauchy-Poisson problem, Dinamika Splošn. Sredy, 18 (1974), 104-210 [Russian].

[3] Okamoto, H., Stationary free boundary problems for circular flows with or without surface tension, Lecture Notes in Num. Appl. Anal., 5 (1982), 233-251.

[4] - Nonstationary or stationary free boundary problems for perfect fluid with surface tension, Lecture Notes in Num. Appl. Anal., 6 (1983), 143-154.

[5] Bifurcation phenomena in a free boundary problem for a circulating flow with surface tension, Math. Meth. Appl. Sci., 6 (1984), 215-233.

[6] - Nonstationary free boundary problem for perfect fluid with surface tension, J. Math. Soc. Japan, 38 (1986), 381-401.

[7] Yosihara, H., Gravity waves on the free surface of an incompressible perfect fluid of finite depth, Publ. RIMS, Kyoto Univ., 18 (1982), 49-96. 
